University of Wollongong

Research Online

Faculty of Engineering and Information

Faculty of Engineering and Information

Sciences - Papers: Part A

Sciences

$1-1-2013$

\title{
The local index formula in noncommutative geometry revisited
}

Alan L. Carey

Australian National University

John Phillips

University of Victoria

Adam C. Rennie

Australian National University, renniea@uow.edu.au

Fyodor A. Sukochev

Flinders University

Follow this and additional works at: https://ro.uow.edu.au/eispapers

Part of the Engineering Commons, and the Science and Technology Studies Commons

Research Online is the open access institutional repository for the University of Wollongong. For further information contact the UOW Library: research-pubs@uow.edu.au 


\title{
The local index formula in noncommutative geometry revisited
}

\author{
Abstract \\ In this review we discuss the local index formula in noncommutative geomety from the viewpoint of two \\ new proofs are partly inspired by the approach of Higson especially that in but they differ in several \\ fundamental aspedcts, in particular they apply to semifinite spectral triples for a *s-subalgebra A of a \\ general semifinite von Neumann algebra. Our proofs are novel even in the setting of the original theorem \\ and reduce the hypotheses of the theorem to those necessary for its statement. \\ These proofs rely on the introduction of a function valued cocycle which is 'almost' a (b, B)-cocycle in the \\ cyclic cohomology of A. They do not need the 'discrete dimension spectrum' assumption of jthe original \\ Connes-Moscovici proof only a much weaker condition on the analytic continuation of certain zeta \\ functions, and this only for part of the statement. \\ In this article we also explain the relationship of the pairing between k-theory amd semifinite spectral \\ triples to KK-theory and the Kasparov product. This discussion shows that simifinite spectral triples are a \\ specific kind of representative of a K K-class and the analytically defined index is compatible with the \\ Kasparov product.

\section{Keywords} \\ local, geometry, revisited, noncommutative, formula, index

\section{Disciplines} \\ Engineering | Science and Technology Studies \\ Publication Details \\ Rennie, A. C., Carey, A. L., Phillips, J. \& Sukochev, F. A. (2013). The local index formula in noncommutative \\ geometry revisited. In G. Dito, M. Kotani, Y. Maeda, H. Moriyoshi \& T. Natsume (Eds.), Noncommutative \\ Geometry and Physics (pp. 3-36). Singapore: World Scientific.
}




\title{
The Local Index Formula in Noncommutative Geometry Revisited
}

\author{
Alan L. Carey ${ }^{1}$, John Phillips ${ }^{2}$, Adam Rennie ${ }^{3}$, Fedor A. Sukochev ${ }^{4}$ \\ 1 Mathematical Sciences Institute, Australian National University, Canberra, \\ ACT. 0200, AUSTRALIA, e-mail: alan.carey@anu.edu.au \\ 2 Department of Mathematics and Statistics, University of Victoria,Victoria, \\ B.C. V8W 3P4, CANADA, ${ }^{\star \star}$ e-mail: phillips@math.uvic.ca \\ 3 Mathematical Sciences Institute, Australian National University, Canberra, \\ ACT. 0200, AUSTRALIA, e-mail: adam.rennie@anu.edu.au \\ 4 School of Mathematics and Statistics, University of New South Wales, \\ Kensington, NSW, Australia, e-mail: f.sukochev@unsw.edu.au
}

All authors were supported by grants from ARC (Australia) and NSERC (Canada).

Abstract

In this review ${ }^{\mathrm{a}}$ we discuss the local index formula in noncommutative geometry (NCG) from the viewpoint of two new proofs that are given in $[16,17]$ and [18] respectively. These proofs are partly inspired by the approach of Higson [35], especially that in [18], but they differ in several fundamental aspects, in particular they apply to semifinite spectral triples for a $*$-subalgebra $\mathcal{A}$ of a general semifinite von Neumann algebra. Our proofs are novel even in the setting of the original theorem, and reduce the hypotheses of the theorem to those necessary for its statement.

These proofs rely on the introduction of a function valued cocycle which is 'almost' a $(b, B)$-cocycle in the cyclic cohomology of $\mathcal{A}$. They do not need the 'discrete dimension spectrum' assumption of the original Connes-Moscovici proof [25], only a much weaker condition on the analytic continuation of certain zeta functions, and this only for part of the statement.

In this article we also explain the relationship of the pairing between $K$ theory and semifinite spectral triples to $K K$-theory and the Kasparov product. This discussion shows that semifinite spectral triples are a specific kind of representative of a $K K$-class, and the analytically defined index is compatible with the Kasparov product.

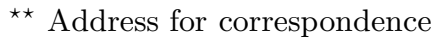

${ }^{a}$ AMS Subject classification: Primary: 19K56, 46L80; secondary: 58B30, 46L87. Keywords and Phrases: von Neumann algebra, Fredholm module, cyclic cohomology, chern character, spectral flow.
} 


\section{INTRODUCTION}

1.1. The motivation for semifinite NCG. The first indication that semifinite theory is natural for NCG comes from the paper [24]. There Connes and Cuntz show that cyclic $n$-cocycles for an appropriate algebra $A$ are in oneto-one correspondence with traces on a certain ideal $J^{n}$ in the free product $A * A$. Assuming some positivity for this trace yields the same kind of Kasparov modules and semifinite Fredholm modules as are described in [37]. In other words, to realise all the cyclic cocycles for an algebra will, in general, necessitate considering semifinite Fredholm modules. The usual approach to the construction of these Fredholm modules is to use semifinite spectral triples.

It is also a natural question to ask how Atiyah's $L^{2}$ index theorem fits into the framework of NCG. While we do not answer this question directly here the results of $[2,49]$ make it clear that this result is within the range of NCG methods as a result of the semifinite version of the local index formula $[16,17]$.

We could also ask what NCG has to do with other results such as the index theorem of Coburn, Douglas, Schaeffer and Singer [22, 31] for Wiener-Hopf operators with almost periodic symbol or the index theorems of Lesch [39] and Phillips-Raeburn [48]. An answer was provided by the noncommutative geometry calculation of the index of Toeplitz operators with noncommutative symbol in [12] which interprets the index as computing spectral flow along a certain path of unbounded self-adjoint Breuer-Fredholm operators. (This uses NCG formulae from [9, 10].)

These results in [12] were a primary inspiration for our new approach to the local index formula of Connes and Moscovici in the setting of semifinite von Neumann algebras that we will discuss in this review. A second important motivation comes from a more general program outlined in [3] for developing a theory of 'von Neumann' or 'semifinite spectral triples'. The primary source of examples for [3] is the theory of foliations.

The articles $[13,16,17,18]$ were written with the view to establishing the main tools of NCG in the wider framework of semifinite theory in such a way as to encompass the standard situation as described in [23] and [25]. This review article has been written to provide a summary of semifinite NCG so that recent new applications of this theory are accessible. Applications in a number of different areas are emerging (see for example [30]) including to a kind of index theory for KMS states on $C^{*}$-algebras $[6,7,15,20]$.

\subsection{Why revisit the proof of the local index formula? Our view is} that the natural setting for the theory of spectral triples is not the bounded operators on a Hilbert space with its ideal of compact operators, but the corresponding situation in a general semifinite von Neumann algebra. This view is supported by [24], and we will amplify on this viewpoint later using results from [37]. 
However, to make spectral triples truly relevant to a semifinite extension of noncommutative geometry, it is necessary to prove the local index formula in this context. The two papers, $[16,17]$ respectively for odd and even semifinite spectral triples, succeed in achieving this extension of the original ConnesMoscovici theorem.

The strategy of the proofs presented in $[16,17]$ are not a semifinite generalisation of [25]. First, for semifinite NCG, we wanted to avoid the discrete dimension spectrum hypothesis of [25]. We were of the view that this hypothesis may be quite hard to check in semifinite, or even type I, spectral triples. This forced us to avoid the starting point of [25], the JLO cocycle [36]. It was [35] that illustrated a possible different approach introducing a kind of 'resolvent cocycle' as an alternative. Unfortunately the cocycle in [35] does not resolve all of the difficulties presented by starting with the JLO formula and still retains the discrete dimension spectrum assumption.

These considerations led us in $[16,17]$ to our first new proof of the local index formula. The argument is different in the odd and even cases relying respectively on a formula for spectral flow presented in [10] and on a generalised McKean-Singer formula proved in [17]. Starting from these formulae we derived a new cyclic cocycle, which we termed the 'resolvent cocycle'. It is, in a sense, a cocycle in the $(b, B)$ version of cyclic cohomology. It provides a substitute for the starting point of [25] (the JLO formula) and it may be used to express the relevant numerical index pairing between the spectral triple, regarded as a 'semifinite $K$-homology class' (as we show, actually a $K K$-class) for an algebra $\mathcal{A}$, and the $K$-theory of $\mathcal{A}$.

Another way to relate our resolvent cocycle to index theory which is suggested by [35] is to homotopy the resolvent cocycle to the Chern character in semifinite NCG. This is the basis of the second new proof of the semifinite local index formula that is contained in [18]. Both of these new proofs avoid the discrete dimension spectrum assumption of [25] replacing it by the minimal assumptions on the singularity structure of the zeta functions that are needed to produce the residue cocycle. It seems highly likely that these minimal assumptions are much easier to check in examples.

\subsection{The $K$-theoretic setting for the semifinite local index formula.}

We remark that semifinite Kasparov modules and semifinite spectral triples provide information that is different from that of the standard theory [23]. We provide later in this article a summary of [37] where it is shown that a semifinite spectral triple for $\mathcal{A}$ represents an element of $K K^{*}(\mathcal{A}, \mathcal{B})$, where $\mathcal{B}$ is the separable norm closed subalgebra of the compact operators in $\mathcal{N}$ generated by the resolvent of $\mathcal{D}$ and the commutators $\left[F_{\mathcal{D}}, a\right]$ for $a \in \mathcal{A}$ where $F_{\mathcal{D}}=\mathcal{D}\left(1+\mathcal{D}^{2}\right)^{-1 / 2}$. This Kasparov module picture is the one implied by $[24]$.

Necessarily, as the local index formula relies on a finite summability hypothesis, we have to consider finitely summable spectral triples $(\mathcal{A}, \mathcal{H}, \mathcal{D})$ with 
spectral dimension $q$. That is, for all $n>q,\left(1+\mathcal{D}^{2}\right)^{-n / 2}$ is trace class in $\mathcal{N}$ and $q$ is the least positive real number for which this is true.

Acknowledgements. We thank Nigel Higson for discussions on his approach to the local index formula and in particular on his point of view of the pseudodifferential calculus. Thanks also to our collaborators Jens Kaad, Ryszard Nest, David Pask and Aidan Sims.

\section{Definitions And BaCkground}

2.1. Semifinite spectral triples. We begin now with some semifinite versions of standard definitions and results. Let $\mathcal{K}_{\mathcal{N}}$ be the $\tau$-compact operators in $\mathcal{N}$ (that is the norm closed ideal generated by the projections $E \in \mathcal{N}$ with $\tau(E)<\infty)$. Here $\tau$ is a fixed faithful, normal, semifinite trace on the von Neumann algebra $\mathcal{N}$.

Definition 2.1. An odd semifinite spectral triple $(\mathcal{A}, \mathcal{H}, \mathcal{D})$ is given by a Hilbert space $\mathcal{H}$, a *algebra $\mathcal{A} \subset \mathcal{N}$ where $\mathcal{N}$ is a semifinite von Neumann algebra acting on $\mathcal{H}$, and a densely defined unbounded self-adjoint operator $\mathcal{D}$ affiliated to $\mathcal{N}$ such that

1) $[\mathcal{D}, a]$ is densely defined and extends to a bounded operator for all $a \in \mathcal{A}$

2) $(\lambda-\mathcal{D})^{-1} \in \mathcal{K}_{\mathcal{N}}$ for all $\lambda \notin \mathbb{R}$

An even semifinite spectral triple $(\mathcal{A}, \mathcal{H}, \mathcal{D})$ has a grading $\gamma \in \mathcal{N}$ such that $\gamma^{*}=\gamma, \gamma^{2}=1, a \gamma=\gamma a$ for all $a \in \mathcal{A}$ and $\mathcal{D} \gamma+\gamma \mathcal{D}=0$

Note that a calligraphic $\mathcal{D}$ will always denote an unbounded self-adjoint operator forming part of a semifinite spectral triple $(\mathcal{A}, \mathcal{H}, \mathcal{D})$.

Definition 2.2. A semifinite spectral triple $(\mathcal{A}, \mathcal{H}, \mathcal{D})$ is $Q C^{k}$ for $k \geq 1$ (Q for quantum) if for all $a \in \mathcal{A}$ the operators a and $[\mathcal{D}, a]$ are in the domain of $\delta^{k}$, where $\delta(T)=[|\mathcal{D}|, T]$ is the partial derivation on $\mathcal{N}$ defined by $|\mathcal{D}|$. We say that $(\mathcal{A}, \mathcal{H}, \mathcal{D})$ is $Q C^{\infty}$ if it is $Q C^{k}$ for all $k \geq 1$.

2.2. Various preliminary remarks. (i) The notation above is meant to be analogous to the classical case, but we introduce the $Q$ so that there is no confusion between the noncommutative case and classical differentiability of functions.

(ii) In this paper, for simplicity of exposition, we will deal only with unital algebras $\mathcal{A} \subset \mathcal{N}$ where the identity of $\mathcal{A}$ is that of $\mathcal{N}$. Henceforth we omit the term semifinite as it is implied by the use of a faithful normal semifinite trace $\tau$ on $\mathcal{N}$ in all of the subsequent text.

(iii) By partial derivation we mean that $\delta$ is defined on some subalgebra of $\mathcal{N}$ which need not be (weakly) dense in $\mathcal{N}$. More precisely, $\operatorname{dom}(\delta)=$ $\{T \in \mathcal{N}: \delta(T)$ is bounded $\}$. We also note that if $T \in \mathcal{N}$, one can show that $[|\mathcal{D}|, T]$ is bounded if and only if $\left[\left(1+\mathcal{D}^{2}\right)^{1 / 2}, T\right]$ is bounded, by using the functional calculus to show that $|\mathcal{D}|-\left(1+\mathcal{D}^{2}\right)^{1 / 2}$ extends to a bounded 
operator in $\mathcal{N}$. In fact, writing $|\mathcal{D}|_{1}=\left(1+\mathcal{D}^{2}\right)^{1 / 2}$ and $\delta_{1}(T)=\left[|\mathcal{D}|_{1}, T\right]$ we have $\operatorname{dom}\left(\delta^{n}\right)=\operatorname{dom}\left(\delta_{1}^{n}\right)$ for all $n$.

Proof. Let $f(\mathcal{D})=\left(1+\mathcal{D}^{2}\right)^{1 / 2}-|\mathcal{D}|$, so, as noted above, $f(\mathcal{D})$ extends to a bounded operator in $\mathcal{N}$. Since

$$
\delta_{1}(T)-\delta(T)=[f(\mathcal{D}), T]
$$

is always bounded, $\operatorname{dom}(\delta)=\operatorname{dom}\left(\delta_{1}\right)$. Now $\delta \delta_{1}=\delta_{1} \delta$, so

$$
\begin{aligned}
\delta_{1}^{2}(T)-\delta^{2}(T) & =\delta_{1}\left(\delta_{1}(T)\right)-\delta_{1}(\delta(T))+\delta_{1}(\delta(T))-\delta(\delta(T)) \\
& =\left[f(\mathcal{D}), \delta_{1}(T)\right]+[f(\mathcal{D}), \delta(T)] .
\end{aligned}
$$

Both terms on the right hand side are bounded, so $\operatorname{dom}\left(\delta^{2}\right)=\operatorname{dom}\left(\delta_{1}^{2}\right)$. The proof proceeds by induction.

Thus the condition defining $Q C^{\infty}$ can be replaced by

$$
a,[\mathcal{D}, a] \in \bigcap_{n \geq 0} \operatorname{dom}\left(\delta_{1}^{n}\right) \quad \forall a \in \mathcal{A} .
$$

This is important as we wish to avoid having to assume that $|\mathcal{D}|$ is invertible.

(iv) If $(\mathcal{A}, \mathcal{H}, \mathcal{D})$ is a $Q C^{\infty}$ spectral triple, we may endow the algebra $\mathcal{A}$ with the topology determined by the seminorms

$$
a \longrightarrow\left\|\delta^{k}(a)\right\|+\left\|\delta^{k}([\mathcal{D}, a])\right\|, \quad k=0,1,2, \ldots
$$

We call this topology the $\delta$-topology and observe that by [50, Lemma 16] we may, without loss of generality, suppose that $\mathcal{A}$ is complete in the $\delta$-topology by completing if necessary. This completion is Fréchet and stable under the holomorphic functional calculus, so we have a sensible spectral theory and $K_{*}(\mathcal{A}) \cong K_{*}(\overline{\mathcal{A}})$ via inclusion, where $\overline{\mathcal{A}}$ is the $C^{*}$-completion of $\mathcal{A}$.

(v) Next we observe that if $T \in \mathcal{N}$ and $[\mathcal{D}, T]$ is bounded, then $[\mathcal{D}, T] \in \mathcal{N}$.

Proof. Observe that $\mathcal{D}$ is affiliated with $\mathcal{N}$, and so commutes with all projections in the commutant of $\mathcal{N}$, and the commutant of $\mathcal{N}$ preserves the domain of $\mathcal{D}$. Thus if $[\mathcal{D}, T]$ is bounded, it too commutes with all projections in the commutant of $\mathcal{N}$, and these projections preserve the domain of $\mathcal{D}$, and so $[\mathcal{D}, T] \in \mathcal{N}$.

Similarly $[|\mathcal{D}|, T],\left[\left(1+\mathcal{D}^{2}\right)^{1 / 2}, T\right]$ and products of the form $\left[\mathcal{D}^{2}, T\right]\left(1+\mathcal{D}^{2}\right)^{-1 / 2}$ that we will encounter later all lie in $\mathcal{N}$.

Definition 2.3. Recall from [32] that if $S \in \mathcal{N}$, the t-th generalized singular value of $S$ for each real $t>0$ is given by

$$
\mu_{t}(S)=\inf \{\|S E\| \mid E \text { is a projection in } \mathcal{N} \text { with } \tau(1-E) \leq t\} .
$$

The ideal $\mathcal{L}^{1}(\mathcal{N})$ consists of those operators $T \in \mathcal{N}$ such that $\|T\|_{1}:=\tau(|T|)<$ $\infty$ where $|T|=\sqrt{T^{*} T}$. In the Type I setting this is the usual trace class ideal. We will simply write $\mathcal{L}^{1}$ for this ideal in order to simplify the notation, and 
denote the norm on $\mathcal{L}^{1}$ by $\|\cdot\|_{1}$. An alternative definition in terms of singular values is that $T \in \mathcal{L}^{1}$ if $\|T\|_{1}:=\int_{0}^{\infty} \mu_{t}(T) d t<\infty$.

Note that in the case where $\mathcal{N} \neq \mathcal{B}(\mathcal{H}), \mathcal{L}^{1}$ need not be complete in this norm but it is complete in the norm $\|\cdot\|_{1}+\|\cdot\|_{\infty}$ (where $\|.\|_{\infty}$ is the uniform norm).

2.3. Hypotheses on the zeta functions. If our spectral triple is finitely summable then this gives us a half-plane where the function

$$
z \mapsto \tau\left(\left(1+\mathcal{D}^{2}\right)^{-z}\right)
$$

is well-defined and holomorphic. If $(\mathcal{A}, \mathcal{H}, \mathcal{D})$ is a $Q C^{\infty}$ spectral triple and $T \in$ $\mathcal{N}$, we write $T^{(n)}$ to denote the iterated commutator $\left[\mathcal{D}^{2},\left[\mathcal{D}^{2},\left[\cdots,\left[\mathcal{D}^{2}, T\right] \cdots\right]\right]\right]$ where we have $n$ commutators with $\mathcal{D}^{2}$. It follows from the remarks after Definition 2.2 that operators of the form $T_{1}^{\left(n_{1}\right)} \cdots T_{k}^{\left(n_{k}\right)}\left(1+\mathcal{D}^{2}\right)^{-\left(n_{1}+\cdots+n_{k}\right) / 2}$ are in $\mathcal{N}$ when $T_{i}=\left[\mathcal{D}, a_{i}\right]$, or $=a_{i}$ for $a_{i} \in \mathcal{A}$.

Our replacement for the hypothesis of discrete dimension spectrum is the following.

Definition 2.4. If $(\mathcal{A}, \mathcal{H}, \mathcal{D})$ is a $Q C^{\infty}$ finitely summable spectral triple, we call

$$
q=\inf \left\{s \in \mathbb{R}: \tau\left(\left(1+\mathcal{D}^{2}\right)^{-s / 2}\right)<\infty\right\}
$$

the spectral dimension of $(\mathcal{A}, \mathcal{H}, \mathcal{D})$. We say that $(\mathcal{A}, \mathcal{H}, \mathcal{D})$ has isolated spectral dimension if for $b$ of the form

$$
b=a_{0}\left[\mathcal{D}, a_{1}\right]^{\left(k_{1}\right)} \cdots\left[\mathcal{D}, a_{m}\right]^{\left(k_{m}\right)}\left(1+\mathcal{D}^{2}\right)^{-m / 2-|k|}
$$

the zeta functions

$$
\zeta_{b}(z-(1-q) / 2)=\tau\left(b\left(1+\mathcal{D}^{2}\right)^{-z+(1-q) / 2}\right)
$$

have analytic continuations to a deleted neighbourhood of $z=(1-q) / 2$.

Observe that we allow the possibility that the analytic continuations of these zeta functions may have an essential singularity at $z=(1-q) / 2$. All that is necessary for us is that the residues at this point exist.

In $[29,25]$, a stronger condition was imposed in order to prove the local index formula. This condition not only specifies a half-plane where the function in (1) is holomorphic, but also that this function analytically continues to $\mathbb{C}$ minus some discrete set. We clarify this in the following definition.

Definition 2.5. Let $(\mathcal{A}, \mathcal{H}, \mathcal{D})$ be a $Q C^{\infty}$ spectral triple. The algebra $\mathcal{B}(\mathcal{A}) \subseteq$ $\mathcal{N}$ is the algebra of polynomials generated by $\delta^{n}(a)$ and $\delta^{n}([\mathcal{D}, a])$ for a $\in$ $\mathcal{A}$ and $n \geq 0$. A $Q C^{\infty}$ spectral triple $(\mathcal{A}, \mathcal{H}, \mathcal{D})$ has discrete dimension spectrum $S d \subseteq \mathbb{C}$ if $S d$ is a discrete set and for all $b \in \mathcal{B}(\mathcal{A})$ the function $\tau\left(b\left(1+\mathcal{D}^{2}\right)^{-z}\right)$ is defined and holomorphic for $\operatorname{Re}(z)$ large, and analytically continues to $\mathbb{C} \backslash S d$. We say the dimension spectrum is simple if this zeta function has poles of order at most one for all $b \in \mathcal{B}(\mathcal{A})$, finite if there is a 
$k \in \mathbb{N}$ such that the function has poles of order at most $k$ for all $b \in \mathcal{B}(\mathcal{A})$ and infinite, if it is not finite.

This discrete dimension spectrum assumption is essential for the proof of the version of the local index formula stated in [25]. In this paper we employ the weaker notion of isolated spectral dimension that is implied by the discrete dimension spectrum assumption.

\section{Spectral Flow}

3.1. The definition of analytic spectral flow. We begin with a discussion of some background from $[2,17,46,47]$. Let $\pi: \mathcal{N} \rightarrow \mathcal{N} / \mathcal{K}_{\mathcal{N}}$ be the canonical mapping. A Breuer-Fredholm operator is one that maps to an invertible operator under $\pi$, [48]. In the Appendix to [48], the theory of Breuer-Fredholm operators for the case where $\mathcal{N}$ is not a factor is developed in analogy with the factor case of Breuer, $[4,5]$. In [17] this theory was developed further to handle the situations encountered in the proof of the local index formula. We will review this theory in Section 4.

As usual $D$ is an unbounded densely defined self-adjoint Breuer-Fredholm operator on $\mathcal{H}$ (meaning $D\left(1+D^{2}\right)^{-1 / 2}$ is bounded and Breuer-Fredholm in $\mathcal{N})$ with $\left(1+D^{2}\right)^{-1 / 2} \in \mathcal{K}_{\mathcal{N}}$. For a unitary $u \in \mathcal{N}$ such that $[D, u]$ is a bounded operator, the path

$$
D_{t}^{u}:=(1-t) D+t u D u^{*}
$$

of unbounded self-adjoint Breuer-Fredholm operators is continuous in the sense that

$$
F_{t}^{u}:=D_{t}^{u}\left(1+\left(D_{t}^{u}\right)^{2}\right)^{-\frac{1}{2}}
$$

is a norm continuous path of self-adjoint Breuer-Fredholm operators in $\mathcal{N}$ [9]. Recall that the Breuer-Fredholm index of a Breuer-Fredholm operator $T$ is defined by

$$
\operatorname{Index}(T)=\tau\left(Q_{\text {ker } T}\right)-\tau\left(Q_{\operatorname{coker} T}\right)
$$

where $Q_{\text {kerT }}$ and $Q_{\text {cokerT }}$ are the projections onto the kernel and cokernel of $T$.

Definition 3.1. If $\left\{F_{t}\right\}$ is a continuous path of self-adjoint Breuer-Fredholm operators in $\mathcal{N}$, then the definition of the spectral flow of the path, $s f\left(\left\{F_{t}\right\}\right)$ is based on the following sequence of observations in [44]:

1. The function $t \mapsto \operatorname{sign}\left(F_{t}\right)$ is typically discontinuous as is the projectionvalued mapping $t \mapsto P_{t}=\frac{1}{2}\left(\operatorname{sign}\left(F_{t}\right)+1\right)$. However $t \mapsto \pi\left(P_{t}\right)$ is continuous. 2. If $P$ and $Q$ are projections in $\mathcal{N}$ and $\|\pi(P)-\pi(Q)\|<1$ then $P Q: Q \mathcal{H} \rightarrow$ $P \mathcal{H}$ is a Breuer-Fredholm operator and so $\operatorname{Index}(P Q) \in \mathbb{R}$ is well-defined (see Lemma 4.1 of [2]). 
3. If we partition the parameter interval of $\left\{F_{t}\right\}$ so that the $\pi\left(P_{t}\right)$ do not vary much in norm on each subinterval of the partition then

$$
s f\left(\left\{F_{t}\right\}\right):=\sum_{i=1}^{n} \operatorname{Index}\left(P_{t_{i-1}} P_{t_{i}}\right)
$$

is a well-defined and (path-) homotopy-invariant number which agrees with the usual notion of spectral flow in the type $I_{\infty}$ case.

4. For $D$ and $u$ as above, we define the spectral flow of the path $D_{t}^{u}:=(1-$ t) $D+t u D u^{*}$ to be the spectral flow of the path $F_{t}$ where $F_{t}=D_{t}^{u}\left(1+\left(D_{t}^{u}\right)^{2}\right)^{-\frac{1}{2}}$. We denote this by

$$
s f\left(D, u D u^{*}\right)=s f\left(\left\{F_{t}\right\}\right),
$$

and observe that this is an integer in the $\mathcal{N}=\mathcal{B}(\mathcal{H})$ case and a real number in the general semifinite case.

Special cases of spectral flow in a semifinite von Neumann algebra were discussed in $[41,44,45]$.

Let $P$ denote the projection onto the nonnegative spectral subspace of $D$. The spectral flow along $\left\{D_{t}^{u}\right\}$ is equal to $s f\left(\left\{F_{t}\right\}\right)$ and by [9] this is the Breuer-Fredholm index of $P u P u^{*}$. (Note that $\operatorname{sign} F_{1}^{u}=2 u P u^{*}-1$ and that for this special path we have $P-u P u^{*}$ is compact so $P u P u^{*}$ is certainly Breuer-Fredholm from $u P u^{*} \mathcal{H} \rightarrow P \mathcal{H}$.) Now, [48, Appendix B], we have $\operatorname{Index}\left(P u P u^{*}\right)=\operatorname{Index}(P u P)$.

3.2. Spectral Flow Formulae. We now introduce the spectral flow formula of $[9,10]$ which is the starting point for our first new proof of the local index formula in the odd case. We start with a semifinite spectral triple $(\mathcal{A}, \mathcal{H}, \mathcal{D})$ and aim to compute the spectral flow from $\mathcal{D}$ to $u \mathcal{D} u^{*}$, where $u \in \mathcal{A}$ is unitary with $[\mathcal{D}, u]$ bounded, in the situation where $(\mathcal{A}, \mathcal{H}, \mathcal{D})$ has spectral dimension $q \geq 1$. Thus for any $n>q$ we have by Theorem 9.3 of [10]:

$$
s f\left(\mathcal{D}, u \mathcal{D} u^{*}\right)=\frac{1}{C_{n / 2}} \int_{0}^{1} \tau\left(u\left[\mathcal{D}, u^{*}\right]\left(1+\left(\mathcal{D}+t u\left[\mathcal{D}, u^{*}\right]\right)^{2}\right)^{-n / 2}\right) d t,
$$

with $C_{n / 2}=\int_{-\infty}^{\infty}\left(1+x^{2}\right)^{-n / 2} d x$. This real number $s f\left(\mathcal{D}, u \mathcal{D} u^{*}\right)$ recovers the pairing of the $K$-homology class $[\mathcal{D}]$ of $\mathcal{A}$ with the $K_{1}(\mathcal{A})$ class $[u]$ (see below).There is a geometric way to view this formula. It is shown in [10] that for the functional $X \mapsto \tau\left(X\left(1+(\mathcal{D}+X)^{2}\right)^{-n / 2}\right), X \in \mathcal{N}_{s a}$, determines an exact one-form on $\mathcal{N}_{s a}$, the tangent space to the affine Banach manifold $\mathcal{D}+\mathcal{N}_{s a}$ at the point $\mathcal{D}+Y \in \mathcal{D}+\mathcal{N}_{s a}$. Thus (2) represents the integral of this one-form along the path $\left\{\mathcal{D}_{t}=(1-t) \mathcal{D}+t u \mathcal{D} u^{*}\right\}$ provided one appreciates that $\dot{\mathcal{D}}_{t}=u\left[\mathcal{D}, u^{*}\right]$ is a tangent vector to this path. Moreover this formula is scale invariant. By this we mean that if we replace $\mathcal{D}$ by $\epsilon \mathcal{D}$, for $\epsilon>0$, in the right hand side of (2), then the left hand side is unchanged, since spectral flow is invariant with respect to change of scale. This fact is important in the proof. 
3.3. Relation to Cyclic Cohomology. One can also interpret spectral flow (in the type I case) as the pairing between an odd $K$-theory class represented by a unitary $u$, and an odd $K$-homology class represented by $(\mathcal{A}, \mathcal{H}, \mathcal{D}),[23$, Chapter III,IV]. This point of view also makes sense in the general semifinite setting, though one must suitably interpret $K$-homology; see the discussion in Section 5. A central feature of [23] is the translation of the $K$-theory pairing to cyclic theory in order to obtain index theorems. One associates to a suitable representative of a $K$-theory class, respectively a $K$-homology class, a class in periodic cyclic homology, respectively a class in periodic cyclic cohomology, called a Chern character in both cases. The principal result is then

$$
s f\left(\mathcal{D}, u \mathcal{D} u^{*}\right)=\langle[u],[(\mathcal{A}, \mathcal{H}, \mathcal{D})]\rangle=-\frac{1}{\sqrt{2 \pi i}}\left\langle\left[C h_{*}(u)\right],\left[C h^{*}(\mathcal{A}, \mathcal{H}, \mathcal{D})\right]\right\rangle,
$$

where $[u] \in K_{1}(\mathcal{A})$ is a $K$-theory class with representative $u$ and $[(\mathcal{A}, \mathcal{H}, \mathcal{D})]$ is the $K$-homology class of the spectral triple $(\mathcal{A}, \mathcal{H}, \mathcal{D})$ (again we refer to Section 5 for the meaning of $K$-homology class in this context).

On the right hand side, $C h_{*}(u)$ is the Chern character of $u$, and $\left[C h_{*}(u)\right]$ its periodic cyclic homology class. Similarly $\left[C h^{*}(\mathcal{A}, \mathcal{H}, \mathcal{D})\right]$ is the periodic cyclic cohomology class of the Chern character of $(\mathcal{A}, \mathcal{H}, \mathcal{D})$. The analogue of Equation (3), for a suitable cocycle associated to $(\mathcal{A}, \mathcal{H}, \mathcal{D})$, in the general semifinite case is part of our main result. A similar result holds for the pairing of classes of projections in $K_{0}(\mathcal{A})$ and even spectral triples.

We will use the normalised $(b, B)$-bicomplex (see $[23,40])$. We introduce the following linear spaces. Let $C_{m}=\mathcal{A} \otimes \overline{\mathcal{A}}^{\otimes m}$ where $\overline{\mathcal{A}}$ is the quotient $\mathcal{A} / \mathbb{C} I$ with $I$ being the identity element of $\mathcal{A}$ and (assuming with no loss of generality that $\mathcal{A}$ is complete in the $\delta$-topology) we employ the projective tensor product. Let $C^{m}=\operatorname{Hom}\left(C_{m}, \mathbb{C}\right)$ be the linear space of continuous multilinear functionals on $C_{m}$. We may define the $(b, B)$ bicomplex using these spaces (as opposed to using $C_{m}=\mathcal{A}^{\otimes m+1}$ et cetera) and the resulting cohomology will be the same. This follows because the bicomplex defined using $\mathcal{A} \otimes \overline{\mathcal{A}}^{\otimes m}$ is quasi-isomorphic to that defined using $\mathcal{A} \otimes \mathcal{A}^{\otimes m}$.

A normalised (b, B)-cochain, $\phi$ is a finite collection of continuous multilinear functionals on $\mathcal{A}$,

$$
\phi=\left\{\phi_{m}\right\}_{m=1,2, \ldots, M} \text { with } \phi_{m} \in C^{m} .
$$

It is a (normalised) (b, B)-cocycle if, for all $m, b \phi_{m}+B \phi_{m+2}=0$ where $b: C^{m} \rightarrow C^{m+1}, B: C^{m} \rightarrow C^{m-1}$ are the coboundary operators given by

$$
\begin{gathered}
\left(B \phi_{m}\right)\left(a_{0}, a_{1}, \ldots, a_{m-1}\right)=\sum_{j=0}^{m-1}(-1)^{(m-1) j} \phi_{m}\left(1, a_{j}, a_{j+1}, \ldots, a_{m-1}, a_{0}, \ldots, a_{j-1}\right) \\
\left(b \phi_{m-2}\right)\left(a_{0}, a_{1}, \ldots, a_{m-1}\right)= \\
\sum_{j=0}^{m-2}(-1)^{j} \phi_{m-2}\left(a_{0}, a_{1}, \ldots, a_{j} a_{j+1}, \ldots, a_{m-1}\right)+(-1)^{m-1} \phi_{m-2}\left(a_{m-1} a_{0}, a_{1}, \ldots, a_{m-2}\right) .
\end{gathered}
$$


We write $(b+B) \phi=0$ for brevity. Thought of as functionals on $\mathcal{A}^{\otimes m+1}$ a normalised cocycle will satisfy $\phi\left(a_{0}, a_{1}, \ldots, a_{n}\right)=0$ whenever any $a_{j}=1$ for $j \geq 1$. An odd (even) cochain has $\left\{\phi_{m}\right\}=0$ for $m$ even (odd).

Similarly, a $\left(\mathbf{b}^{\mathbf{T}}, \mathbf{B}^{\mathbf{T}}\right)$-chain, $c$ is a (possibly infinite) collection $c=$ $\left\{c_{m}\right\}_{m=1,2, \ldots}$ with $c_{m} \in C_{m}$. The $(b, B)$-chain $\left\{c_{m}\right\}$ is a $\left(\mathbf{b}^{\mathbf{T}}, \mathbf{B}^{\mathbf{T}}\right)$-cycle if $b^{T} c_{m+2}+B^{T} c_{m}=0$ for all $m$. More briefly, we write $\left(b^{T}+B^{T}\right) c=0$. Here $b^{T}, B^{T}$ are the boundary operators of cyclic homology, and are the transpose of the coboundary operators $b, B$ in the following sense.

The pairing between a $(b, B)$-cochain $\phi=\left\{\phi_{m}\right\}_{m=1}^{M}$ and a $\left(b^{T}, B^{T}\right)$-chain $c=\left\{c_{m}\right\}$ is given by

$$
\langle\phi, c\rangle=\sum_{m=1}^{M} \phi_{m}\left(c_{m}\right) .
$$

This pairing satisfies

$$
\langle(b+B) \phi, c\rangle=\left\langle\phi,\left(b^{T}+B^{T}\right) c\right\rangle .
$$

We use this fact in Section 8 in the following way. We call $c=\left(c_{m}\right)_{m}$ odd an odd normalised $\left(\mathbf{b}^{\mathbf{T}}, \mathbf{B}^{\mathbf{T}}\right)$-boundary if there is some even chain $e=$ $\left\{e_{m}\right\}_{m}$ even with $c_{m}=b^{T} e_{m+1}+B^{T} e_{m-1}$ for all $m$. If we pair a normalised $(b, B)$-cocycle $\phi$ with a normalised $\left(b^{T}, B^{T}\right)$-boundary $c$ we find

$$
\langle\phi, c\rangle=\left\langle\phi,\left(b^{T}+B^{T}\right) e\right\rangle=\langle(b+B) \phi, e\rangle=0 .
$$

There is an analogous definition in the case of even chains $c=\left(c_{m}\right)_{m}$ even . All of the cocycles we consider in this paper are in fact defined as functionals on $\oplus_{m} \mathcal{A} \otimes \overline{\mathcal{A}}^{\otimes m}$. Henceforth we will drop the superscript on $b^{T}, B^{T}$ and just write $b, B$ for both boundary and coboundary operators as the meaning will be clear from the context.

We recall that the Chern character $C h_{*}(u)$ of a unitary $u \in \mathcal{A}$ is the following (infinite) collection of odd chains $C h_{2 j+1}(u)$ satisfying $b C h_{2 j+3}(u)+$ $B C h_{2 j+1}(u)=0$,

$$
C h_{2 j+1}(u)=(-1)^{j} j ! u^{*} \otimes u \otimes u^{*} \otimes \cdots \otimes u \quad(2 j+2 \text { entries }) .
$$

In our first proof of the local index formula we find that $u$ and $u^{*}$ enter in a symmetric way into the formulae. This presents no difficulty as

$$
C h_{*}\left(u^{*}\right)+C h_{*}(u)
$$

is homologous to zero in the normalised (entire) $(b, B)$ chain complex, see [16] for a proof.

\section{Fredholm Theory in Semifinite von Neumann Algebras}

4.1. The reason we need a more general theory. In our approach we must study Fredholm operators in a "skew-corner" of our semifinite von Neumann algebra $\mathcal{N}$. That is, if $P$ and $Q$ are projections in $\mathcal{N}$ (not necessarily infinite and not necessarily equivalent) we have to extend the notion of $\tau$ index and $\tau$-Fredholm to operators $T \in P \mathcal{N} Q$. If $\mathcal{N}$ is a factor, this is much 
easier and is done in Appendix A of [47]. We simply refer to them as $(P \cdot Q)$ Fredholm operators. Most results work in this setting; however the ploy used in [47] of invoking the existence of a partial isometry from $P$ to $Q$ to reduce to the case $P \mathcal{N} P$ (solved in [48]) is not available. In fact, because of examples to which our version of the McKean-Singer Theorem applies, $P$ and $Q$ are not generally equivalent.

One notable result that is different in the nonfactor setting (even if $P=Q$ ) is that the set of $(P \cdot Q)$-Fredholm operators with a given index is open but is not generally connected: information is lost when one fixes a trace to obtain a real-valued index. That the set of $(P \cdot Q)$-Fredholm operators with a given index is open (and other facts) is very sensitive to the order in which the expected results are proved. As the Fredholm alternative is not available in the $(P \cdot Q)$ setting, we take a novel approach and deduce many facts from the formula for the index of a product. We also study unbounded operators affiliated to a "skew-corner". The following discussion is a mild paraphrasing of the corresponding discussion in [17].

4.2. Preliminaries. If $T$ is an operator in the von Neumann algebra $\mathcal{N}$ (or $T$ is closed, densely defined, affiliated to $\mathcal{N}$ and $T=P T Q$, in which case we say $T$ is affiliated to $P \mathcal{N} Q$ ) then we let $R_{T}$ and $N_{T}$ be the projections on the closure of the range of $T$ and the kernel of $T$, respectively. If $T \in P \mathcal{N} Q$, (or $T$ is closed and affiliated to $P \mathcal{N} Q$ ) then we will denote the projection on $\operatorname{ker}_{Q}(T)=\operatorname{ker}\left(T_{\left.\right|_{Q(\mathcal{H})}}\right)=\operatorname{ker}(T) \cap Q(\mathcal{H})$ by $N_{T}^{Q}$ and observe that $N_{T}^{Q}=$ $Q N_{T}=N_{T} Q \leq Q$ while $R_{T} \leq P$.

Definition 4.1. With the usual assumptions on $\mathcal{N}$ let $P$ and $Q$ be projections (not necessarily infinite, or equivalent) in $\mathcal{N}$, and let $T \in P \mathcal{N} Q$. Then $T$ is called $(P \cdot Q)$-Fredholm if and only if

(1) $\tau\left(N_{T}^{Q}\right)<\infty$, and $\tau\left(N_{T^{*}}^{P}\right)<\infty$, and

(2) There exists a $\tau$-finite projection $E \leq P$ with $\operatorname{range}(P-E) \subseteq \operatorname{range}(T)$.

If $T$ is $(P \cdot Q)$-Fredholm then the $(P \cdot Q)$-Index of $T$ is

$$
\operatorname{Index}(T)=\tau\left(N_{T}^{Q}\right)-\tau\left(N_{T^{*}}^{P}\right) .
$$

The elementary consequences of this definition we record in the next result.

Lemma 4.2. With the usual assumptions on $\mathcal{N}$, let $T \in P \mathcal{N} Q$. Then,

(1) With $P_{1}=R_{T}$ and $Q_{1}=Q-N_{T}^{Q}=\operatorname{supp}(T)=R_{T^{*}}$, we have that $T$ is $(P \cdot Q)$-Fredholm if and only if $T$ is $\left(P_{1} \cdot Q_{1}\right)$-Fredholm and in this case, the $\left(P_{1} \cdot Q_{1}\right)$-Index of $T$ is 0 , while the $(P \cdot Q)$-Index of $T$ is $\tau\left(Q-Q_{1}\right)-\tau\left(P-P_{1}\right)$.

(2) If $T$ is $(P \cdot Q)$-Fredholm, then $T^{*}$ is $(Q \cdot P)$-Fredholm and $\operatorname{Index}\left(T^{*}\right)=$ - Index $(T)$. If $T=V|T|$ is the polar decomposition, then $V$ is $(P \cdot Q)$ Fredholm with $\operatorname{Index}(V)=\operatorname{Index}(T)$ and $|T|$ is $(Q \cdot Q)$-Fredholm of index 0 . 
(3) If $T=V|T|$ is $(P \cdot Q)$-Fredholm, then there exists a spectral projection $Q_{0} \leq Q$ for $|T|$ so that $\tau\left(Q-Q_{0}\right)<\infty$, and $P_{0}=V Q_{0} V^{*}$ satisfies: $\tau\left(P-P_{0}\right)<\infty, P_{0}(\mathcal{H})=\operatorname{range}\left(T Q_{0}\right) \subset \operatorname{range}(T), Q_{0}(\mathcal{H}) \subset \operatorname{range}\left(T^{*}\right)$, $T Q_{0}=P_{0} T Q_{0}: Q_{0}(\mathcal{H}) \rightarrow P_{0}(\mathcal{H})$ and $T^{*} P_{0}=Q_{0} T^{*} P_{0}: P_{0}(\mathcal{H}) \rightarrow Q_{0}(\mathcal{H})$ are invertible as bounded linear operators.

(4) The set of all $(P \cdot Q)$-Fredholm operators in $P \mathcal{N} Q$ is open in the norm topology.

We will not discuss the proof of this lemma here referring to [17].

4.3. The parametrix. The material in this subsection is not standard and so we discuss it in more detail.

Definition 4.3. If $T \in P \mathcal{N} Q$, then a parametrix for $T$ is an operator $S \in Q \mathcal{N} P$ satisfying $S T=Q+k_{1}$ and $T S=P+k_{2}$ where $k_{1} \in \mathcal{K}_{Q \mathcal{N} Q}$ and $k_{2} \in \mathcal{K}_{P \mathcal{N} P}$.

Lemma 4.4. With the usual assumptions on $\mathcal{N}$, then $T \in P \mathcal{N} Q$ is $(P \cdot Q)$ Fredholm if and only if $T$ has a parametrix $S \in Q \mathcal{N} P$. Moreover, any such parametrix is $(Q \cdot P)$-Fredholm.

Proof. Let $S$ be a parametrix for $T$. Then $T S=P+k_{2}$ is Fredholm in $P \mathcal{N} P$ by Appendix B of [48]. Hence there exists a projection $P_{1} \leq P$ with $\tau\left(P-P_{1}\right)<\infty$ and $P_{1}(\mathcal{H}) \subset \operatorname{range}(T S) \subset \operatorname{range}(T)$. So, $N_{T^{*}}^{P}=P-R_{T} \leq$ $P-P_{1}$ is $\tau$-finite. On the other hand, $T^{*} S^{*}=(S T)^{*}=Q+k_{1}^{*}$ is Fredholm in $Q \mathcal{N} Q$ again by Appendix B of [48] and so by the same $\operatorname{argument} N_{T}^{Q}$ is also $\tau$-finite. That is, $T$ is $(P \cdot Q)$-Fredholm and similarly $S$ is $(Q \cdot P)$-Fredholm.

Now suppose that $T$ is $(P \cdot Q)$-Fredholm. By part (3) of Lemma 4.2, there exist projections $Q_{0}$ and $P_{0}$ which are $\tau$-cofinite in $Q$ and $P$ respectively so that $T Q_{0}=P_{0} T Q_{0}: Q_{0}(\mathcal{H}) \rightarrow P_{0}(\mathcal{H})$ is invertible as a bounded linear operator. Let $S$ be its inverse. Then $S \in \mathcal{N}$ so that $S=Q_{0} S P_{0} \in Q \mathcal{N} P$, and $S T Q_{0}=Q_{0}$ and $T Q_{0} S=P_{0}$. Finally,

$S T=S T Q_{0}+S T\left(Q-Q_{0}\right)=Q_{0}+k=Q+k_{1}$ and $T S=T Q_{0} S=P_{0}=P+k_{2}$, where $k_{1} \in \mathcal{K}_{Q \mathcal{N} Q}$ and $k_{2} \in \mathcal{K}_{P \mathcal{N} P}$. That is, $S$ is a parametrix for $T$.

Lemma 4.5. We retain the usual assumptions on $\mathcal{N}$.

(1) Let $T \in P \mathcal{N} Q$ be $(P \cdot Q)$-Fredholm. If $k \in P \mathcal{K}_{\mathcal{N}} Q$ then $T+k$ is also $(P \cdot Q)$-Fredholm.

(2) If $T \in P \mathcal{N} Q$ is $(P \cdot Q)$-Fredholm and $S \in G \mathcal{N} P$ is $(G \cdot P)$-Fredholm, then $S T$ is $(G \cdot Q)$-Fredholm. 
Proof. One checks that if $S$ is a parametrix for $T$ then $S$ is also a parametrix for $T+k$ and that if $T_{1}$ is a parametrix for $T$ and $S_{1}$ is a parametrix for $S$, then $T_{1} S_{1}$ is a parametrix for $S T$.

Proposition 4.6. Let $G, P, Q$ be projections in $\mathcal{N}$ (with trace $\tau$ ) and let $T \in P \mathcal{N} Q$ be $(P \cdot Q)$-Fredholm and $S \in G \mathcal{N} P$ be $(G \cdot P)$-Fredholm, respectively. Then, $S T$ is $(G \cdot Q)$-Fredholm and

$$
\operatorname{Index}(S T)=\operatorname{Index}(S)+\operatorname{Index}(T) .
$$

The proof of this proposition essentially follows Breuer in [5] although there are changes forced by the greater generality of our discussion. The proof of the Proposition rests on the following:

Lemma 4.7. (Cf. Lemma 1 of [5]) With the hypotheses of the Proposition:

$$
N_{S T}^{Q}-N_{T}^{Q} \sim \inf \left(R_{T}, N_{S}^{P}\right)
$$

Corollary 4.8. (Invariance properties of the $(P \cdot Q)$-Index) Let $T \in P \mathcal{N} Q$.

(1) If $T$ is $(P \cdot Q)$-Fredholm then there exists $\delta>0$ so that if $S \in P \mathcal{N} Q$ and $\|T-S\|<\delta$ then $S$ is $(P \cdot Q)$-Fredholm and $\operatorname{Index}(S)=\operatorname{Index}(T)$.

(2) If $T$ is $(P \cdot Q)$-Fredholm and $k \in P \mathcal{K}_{\mathcal{N}} Q$ then $T+k$ is $(P \cdot Q)$-Fredholm and $\operatorname{Index}(T+k)=\operatorname{Index}(T)$.

We omit the details of the proofs referring again to [17].

4.4. Spectral flow. In [47] spectral flow is defined in a semifinite factor using the index of Breuer-Fredholm operators in a skew-corner $P \mathcal{N} Q$ (in particular the operator $P Q$ ) and uses the product theorem for the index and other standard properties. The non-factor case for Toeplitz operators $(P=Q)$ is covered in [48] but the more subtle "skew-corner" case did not appear in the literature until [17]. The discussion below explains what is needed to extend [47] to the nonfactor setting. This material was required for $[10,12,16]$ although we did not discuss it there. For the McKean-Singer formula we need to generalise some of these results to closed, densely defined operators $T$ affiliated to $\mathcal{N}$ satisfying $T=P T Q$ (in our terminology, $T$ is affiliated to $P \mathcal{N} Q$ ) by studying the map $T \mapsto T\left(1+|T|^{2}\right)^{-1 / 2}$.

Definition 4.9. A closed, densely defined operator $T$ affiliated to $P \mathcal{N} Q$ is $(P \cdot Q)$-Fredholm if

(1) $\tau\left(N_{T}^{Q}\right)<\infty$, and $\tau\left(N_{T^{*}}^{P}\right)<\infty$, and

(2) There exists a $\tau$-finite projection $E \leq P$ with $\operatorname{range}(P-E) \subset \operatorname{range}(T)$.

If $T$ is $(P \cdot Q)$-Fredholm then the $(P \cdot Q)$-Index of $T$ is: $\operatorname{Index}(T)=\tau\left(N_{T}^{Q}\right)-$ $\tau\left(N_{T^{*}}^{P}\right)$. 
Remark. Using the equalities: range $\left(1+|T|^{2}\right)^{-1 / 2}=\operatorname{dom}\left(\left(1+|T|^{2}\right)^{1 / 2}\right)=$ $\operatorname{dom}(|T|)=\operatorname{dom}(T)$ one can show that: $\operatorname{range}(T)=\operatorname{range}\left(T\left(1+|T|^{2}\right)^{-1 / 2}\right)$; $\operatorname{ker}(T)=\operatorname{ker}\left(T\left(1+|T|^{2}\right)^{-1 / 2}\right)$ and $\operatorname{ker}\left(T^{*}\right)=\operatorname{ker}\left(\left[T\left(1+|T|^{2}\right)^{-1 / 2}\right]^{*}\right)$. A little more thought completes the following:

Proposition 4.10. (Index) If $T$ is a closed, densely defined operator affiliated to $P \mathcal{N} Q$, then $T$ is $(P \cdot Q)$-Fredholm if and only if the operator $T\left(1+|T|^{2}\right)^{-1 / 2}$ is $(P \cdot Q)$-Fredholm in $P \mathcal{N} Q$. In this case,

$$
\operatorname{Index}(T)=\operatorname{Index}\left(T\left(1+|T|^{2}\right)^{-1 / 2}\right) \text {. }
$$

The expected properties of these Fredholm operators now follow in a relatively straightforward way, [17].

Proposition 4.11. (i) (Continuity) If $T$ is a closed, densely defined operator affiliated to $P \mathcal{N} Q$, and $A \in P \mathcal{N} Q$ then $T+A$ is also closed, densely defined, and affiliated to $P \mathcal{N} Q$ and

$$
\left\|T\left(1+|T|^{2}\right)^{-1 / 2}-(T+A)\left(1+|T+A|^{2}\right)^{-1 / 2}\right\| \leq\|A\| .
$$

(ii) (Index continuity) If $T$ is affiliated to $P \mathcal{N} Q$ and $T$ is $(P \cdot Q)$-Fredholm then there exists $\epsilon>0$ so that if $A \in P \mathcal{N} Q$ and $\|A\|<\epsilon$, then $T+A$ is $(P \cdot Q)$-Fredholm and

$$
\operatorname{Index}(T+A)=\operatorname{Index}(T) .
$$

(iii) (Compact perturbation) Let $T$ be any closed, densely defined operator affiliated to $P \mathcal{N} Q$.

(1) If $k \in P \mathcal{K}_{\mathcal{N}} Q$, then the difference $T\left(1+|T|^{2}\right)^{-1 / 2}-(T+k)\left(1+|T+k|^{2}\right)^{-1 / 2}$ is in $P \mathcal{K}_{\mathcal{N}} Q$

(2) If $T$ is $(P \cdot Q)$-Fredholm then for all $k \in P \mathcal{K}_{\mathcal{N}} Q, T+k$ is $(P \cdot Q)$-Fredholm and

$$
\operatorname{Index}(T+k)=\operatorname{Index}(T) .
$$

4.5. The unbounded parametrix. For many geometric examples, the following is a useful notion. If $T$ is a closed, densely defined, unbounded operator affiliated to $P \mathcal{N} Q$ then a parametrix for $T$ is a bounded everywhere defined operator $S \in Q \mathcal{N} P$ such that:

(1) $\overline{T S}=P+k_{1}$ for $k_{1} \in P \mathcal{K}_{\mathcal{N}} P$,

(2) $\overline{S T}=Q+k_{2}$ for $k_{2} \in Q \mathcal{K}_{\mathcal{N}} Q$.

Note that, as $T$ is closed and $S$ is bounded, $T S=\overline{T S}$ is everywhere defined and bounded by (1). For example, if $D$ is an unbounded self-adjoint operator and $\left(1+D^{2}\right)^{-1} \in \mathcal{K}_{\mathcal{N}}$ then $D\left(1+D^{2}\right)^{-1}$ is a parametrix for $D$ since $\overline{D\left(1+D^{2}\right)^{-1} D}=D^{2}\left(1+D^{2}\right)^{-1}=1-\left(1+D^{2}\right)^{-1}$.

Lemma 4.12. If $T$ is a closed, densely defined, unbounded operator affiliated to $P \mathcal{N} Q$ then $T$ has a parametrix if and only if $T$ is $(P \cdot Q)$-Fredholm. 
Proof. If $S$ is a parametrix for $T$ then by (1) $T S$ is everywhere defined and Fredholm in $P \mathcal{N} P$. So there exists a projection $E \leq P$ with $\tau(E)<\infty$ and: range $(P-E) \subset \operatorname{range}(T S) \subset \operatorname{range}(T)$. In particular, this implies (since $T S$ is bounded) that $N_{(T S)^{*}}^{P}$ is $\tau$-finite. But $S^{*} T^{*} \subseteq(T S)^{*}$ and so $N_{T^{*}}^{P} \leq N_{(T S)^{*}}^{P}$. That is, $\tau\left(N_{T^{*}}^{P}\right)<\infty$. Now, $\overline{S T}=Q+k_{2}$ is $(Q \cdot Q)$-Fredholm and so has a $\tau$-finite $Q$-kernel. But $N_{T}^{Q} \leq N_{\frac{Q}{S T}}$. That is, $\tau\left(N_{T}^{Q}\right)<\infty$ and $T$ is $(P \cdot Q)$ Fredholm.

If $T=V|T|$ is $(P \cdot Q)$-Fredholm then $|T|\left(1+|T|^{2}\right)^{-1 / 2}$ is bounded and $(Q \cdot Q)$-Fredholm and so has a parametrix $S$ which we can take to be a function of $|T|\left(1+|T|^{2}\right)^{-1 / 2}$. Thus $S$ commutes with $\left(1+|T|^{2}\right)^{-1 / 2}$. One then checks that $\left(1+|T|^{2}\right)^{-1 / 2} S V^{*}$ is a parametrix for $T$.

Remark. In general a parametrix for a genuinely unbounded Fredholm operator is not Fredholm as its range can not contain the range of a cofinite projection. For instance, the parametrix of $-i \frac{d}{d \theta}$ on $L^{2}\left(S^{1}\right)$ is a compact operator, and so certainly not invertible modulo compact operators.

4.6. The generalised McKean-Singer formula. We were somewhat surprised that the following result had not been proved anywhere in the literature.

Theorem 4.13. (McKean-Singer) Let D be an unbounded self-adjoint operator affiliated to the semifinite von Neumann algebra $\mathcal{N}$ (with faithful normal semifinite trace $\tau)$ with $\left(1+D^{2}\right)^{-1} \in \mathcal{K}_{\mathcal{N}}$. Let $\gamma$ be a self-adjoint unitary in $\mathcal{N}$ which anticommutes with $D$. Finally, let $f$ be a continuous even function on $\mathbf{R}$ with $f(0) \neq 0$ and $f(D)$ trace-class. Let $D^{+}=P^{\perp} D P$ where $P=(\gamma+1) / 2$ and $P^{\perp}=1-P$. Then as an operator affiliated to $P^{\perp} \mathcal{N} P$, $D^{+}$is $\left(P^{\perp} \cdot P\right)$-Fredholm and

$$
\operatorname{Index}\left(D^{+}\right)=\frac{1}{f(0)} \tau(\gamma f(D)) \text {. }
$$

Proof. Let $D^{-}=P D P^{\perp}$. Since $\{D, \gamma\}=0$, we see that relative to the decomposition $1=P \oplus P^{\perp}$ :

$$
\begin{gathered}
\gamma=\left(\begin{array}{cc}
1 & 0 \\
0 & -1
\end{array}\right), D=\left(\begin{array}{cc}
0 & D^{-} \\
D^{+} & 0
\end{array}\right) \\
D^{2}=\left(\begin{array}{cc}
D^{-} D^{+} & 0 \\
0 & D^{+} D^{-}
\end{array}\right),|D|=\left(\begin{array}{cc}
\left|D^{+}\right| & 0 \\
0 & \left|D^{-}\right|
\end{array}\right) .
\end{gathered}
$$

We have already observed that $D\left(1+D^{2}\right)^{-1}$ is a parametrix for $D$. But, then:

$$
D\left(1+D^{2}\right)^{-1}=\left(\begin{array}{cc}
0 & D^{-}\left(P^{\perp}+\left|D^{-}\right|^{2}\right)^{-1} \\
D^{+}\left(P+\left|D^{+}\right|^{2}\right)^{-1} & 0
\end{array}\right) .
$$

Hence $D^{-}\left(P^{\perp}+\left|D^{-}\right|^{2}\right)^{-1}$ is a parametrix for $D^{+}$and so $D^{+}$is $\left(P^{\perp} \cdot P\right)$ Fredholm. Let $D^{+}=V\left|D^{+}\right|$be the polar decomposition of $D^{+}$so that 
$D^{-}=D^{+*}=\left|D^{+}\right| V^{*}$. Then $V \in \mathcal{N}$ is a partial isometry with initial space $P_{1}=V^{*} V=\operatorname{supp}\left(D^{+}\right) \leq P$ and final space $Q_{1}=V V^{*}=\operatorname{range}\left(D^{+}\right)^{-}=$ $\operatorname{supp}\left(D^{-}\right) \leq P^{\perp}$. Then, $\operatorname{ker}\left(D^{+}\right)=P_{0}(\mathcal{H})$ as an operator on $P(\mathcal{H})$ where $P_{0}=P-P_{1}$. Similarly, $\operatorname{coker}\left(D^{+}\right)=\operatorname{ker}\left(D^{-}\right)=Q_{0}(\mathcal{H})$ where $Q_{0}=P^{\perp}-Q_{1}$.

Now, $\left|D^{+}\right|^{2}=D^{-} D^{+}=D^{-} D^{-*}=V^{*}\left|D^{-}\right|^{2} V$ so that $\left|D^{+}\right|=V^{*}\left|D^{-}\right| V$ and if $g$ is any bounded continuous function then, $g\left(\left|D^{+}\right|_{P_{P_{1}(\mathcal{H})}}\right)=$ $V^{*} g\left(\left|D^{-}\right|_{Q_{Q_{1}(\mathcal{H})}}\right) V$. But, as an operators on $P(\mathcal{H})$, and respectively, $P^{\perp}(\mathcal{H})$ we have:

$$
\begin{aligned}
& g\left(\left|D^{+}\right|\right)=P_{1} g\left(\left|D^{+}\right|\right) P_{1} \oplus g(0) P_{0}=g\left(\left|D^{+}\right|_{P_{1}(\mathcal{H})}\right) \oplus g(0) P_{0} \text { and } \\
& g\left(\left|D^{-}\right|\right)=Q_{1} g\left(\left|D^{-}\right|\right) Q_{1} \oplus g(0) Q_{0}=g\left(\left|D^{-}\right|_{Q_{1}(\mathcal{H})}\right) \oplus g(0) Q_{0} .
\end{aligned}
$$

Finally, since $f$ is even, we have $f(D)=f(|D|)$ and so:

$$
\begin{aligned}
& \gamma f(D)=\left(\begin{array}{cc}
f\left(\left|D^{+}\right|\right) & 0 \\
0 & -f\left(\left|D^{-}\right|\right)
\end{array}\right) \\
& =\left(\begin{array}{cc}
\left.f\left(\left|D^{+}\right|_{\left.\left.\right|_{P_{1}(\mathcal{H})}\right)}\right) \oplus f(0) P_{0}\right) & 0 \\
0 & -f\left(\left|D^{-}\right|_{Q_{Q_{1}(\mathcal{H})}}\right) \oplus-f(0) Q_{0}
\end{array}\right) \\
& =\left(\begin{array}{cc}
\left.V^{*} f\left(\left|D^{-}\right|_{\left.\right|_{Q_{1}(\mathcal{H})}}\right) V \oplus f(0) P_{0}\right) & 0 \\
0 & -f\left(\left|D^{-}\right|_{Q_{Q_{1}(\mathcal{H})}}\right) \oplus-f(0) Q_{0}
\end{array}\right) . \\
& \tau(\gamma f(D))=f(0) \tau\left(P_{0}\right)-f(0) \tau\left(Q_{0}\right)=f(0) \operatorname{Index}\left(D^{+}\right) .
\end{aligned}
$$

Corollary 4.14. Let $(\mathcal{A}, \mathcal{H}, \mathcal{D})$ be an even spectral triple with grading $\gamma$, $\left(1+\mathcal{D}^{2}\right)^{-1 / 2} \in \mathcal{L}^{n}(\mathcal{N})$ and $p \in \mathcal{A}$, a projection. Then, relative to the decomposition afforded by $\gamma$ as above, we have:

$$
p=\left(\begin{array}{cc}
p^{+} & 0 \\
0 & p^{-}
\end{array}\right) \text {, where } p^{+}=P p P=P p \text { and } p^{-}=P^{\perp} p P^{\perp}=p P^{\perp} .
$$

So, $p \mathcal{D}^{+} p=p P^{\perp} \mathcal{D} P p=p^{-} \mathcal{D} p^{+}$is an operator affiliated to $p^{-} \mathcal{N} p^{+}$we have that $p^{-} \mathcal{D}^{+} p^{+}$is $\left(p^{-} \cdot p^{+}\right)$-Fredholm and for any fixed $a \geq 0$ its $\left(\mathbf{p}^{-} \cdot \mathbf{p}^{+}\right)$-index is given by:

$$
\operatorname{Index}\left(p \mathcal{D}^{+} p\right)=\operatorname{Index}\left(p^{-} \mathcal{D}^{+} p^{+}\right)=(1+a)^{n / 2} \tau\left(\gamma p\left(p+a+(p \mathcal{D} p)^{2}\right)^{-n / 2}\right) .
$$

Proof. In the above version of the McKean-Singer theorem, we replace $\mathcal{A}$ with $p \mathcal{A} p$ which is a unital subalgebra of the semifinite von Neumann algebra $p \mathcal{N} p$. Moreover, the operator $p \mathcal{D} p$ is self-adjoint and affiliated to $p \mathcal{N} p$, and $p \gamma$ is a grading in $p \mathcal{N} p$. One easily checks that

$$
(p \mathcal{D} p)^{+}=p^{-} \mathcal{D}^{+} p^{+} \text {. }
$$

Let $f(x)=\left(1+a+x^{2}\right)^{-n / 2}$. We may apply the McKean-Singer formula once we show that $\left(p+a+(p \mathcal{D} p)^{2}\right)^{-1 / 2} \in \mathcal{L}^{n}(p \mathcal{N} p)$. It suffices to do this for $a=0$ 
since

$$
\left(p+a+(p \mathcal{D} p)^{2}\right)^{-1 / 2} \leq\left(p+(p \mathcal{D} p)^{2}\right)^{-1 / 2} .
$$

Here is the calculation:

$$
\begin{aligned}
& p\left(1+\mathcal{D}^{2}\right)^{-1} p-\left(p+(p \mathcal{D} p)^{2}\right)^{-1} \\
= & p\left[\left(1+\mathcal{D}^{2}\right)^{-1}-\left\{\left(p+(p \mathcal{D} p)^{2}\right)+(1-p)\right\}^{-1}\right] p \\
= & p\left(1+\mathcal{D}^{2}\right)^{-1}\left(\left(p+(p \mathcal{D} p)^{2}\right)+(1-p)-\left(1+\mathcal{D}^{2}\right)\right)\left\{\left(p+(p \mathcal{D} p)^{2}\right)+(1-p)\right\}^{-1} p \\
= & p\left(1+\mathcal{D}^{2}\right)^{-1}\left((p \mathcal{D} p)^{2}-\mathcal{D}^{2}\right) p\left(p+(p \mathcal{D} p)^{2}\right)^{-1} p \\
= & p\left(1+\mathcal{D}^{2}\right)^{-1}([p, \mathcal{D}] p \mathcal{D} p+\mathcal{D} p[\mathcal{D}, p]+\mathcal{D}[p, \mathcal{D}]) p\left(p+(p \mathcal{D} p)^{2}\right)^{-1} p \\
= & p\left(1+\mathcal{D}^{2}\right)^{-1}[p, \mathcal{D}] p \mathcal{D} p\left(p+(p \mathcal{D} p)^{2}\right)^{-1} p \\
& +p\left(1+\mathcal{D}^{2}\right)^{-1} \mathcal{D} p[\mathcal{D}, p] p\left(p+(p \mathcal{D} p)^{2}\right)^{-1} p \\
& +p\left(1+\mathcal{D}^{2}\right)^{-1} \mathcal{D}[p, \mathcal{D}] p\left(p+(p \mathcal{D} p)^{2}\right)^{-1} p .
\end{aligned}
$$

Now, since $\left|\mathcal{D}\left(1+\mathcal{D}^{2}\right)^{-1}\right| \leq\left(1+\mathcal{D}^{2}\right)^{-1 / 2}$, it follows that the three terms in the last equality are in $\mathcal{L}^{n / 2}, \mathcal{L}^{n}$, and $\mathcal{L}^{n}$, respectively, and so their sum is in $\mathcal{L}^{n}$. Since, $p\left(1+\mathcal{D}^{2}\right)^{-1} p \in \mathcal{L}^{n / 2}$, we see from the first line in the displayed equations that $\left(p+(p \mathcal{D} p)^{2}\right)^{-1}$ is in $\mathcal{L}^{n}$.

Now, given this new information, we reconsider the three terms in the last line again, and see that they are in $\mathcal{L}^{n / 2}, \mathcal{L}^{n} \cdot \mathcal{L}^{n}$, and $\mathcal{L}^{n} \cdot \mathcal{L}^{n}$, respectively, and so their sum is in $\mathcal{L}^{n / 2}$. Thus, $\left(p+(p \mathcal{D} p)^{2}\right)^{-1}$ is, in fact, in $\mathcal{L}^{n / 2}$ : in other words, $\left(p+(p \mathcal{D} p)^{2}\right)^{-1 / 2}$ is in $\mathcal{L}^{n}$ as claimed.

From now on, we follow convention and denote the above index by Index $\left(p \mathcal{D}^{+} p\right)$; effectively disguising the fact that $p \mathcal{D}^{+} p$ is, in fact, Fredholm relative to the "skew-corner," $p^{-\mathcal{N} p} p^{+}$.

Remark: The ideal $\mathcal{L}^{n}(\mathcal{N})$ can be replaced by any symmetric ideal $\mathcal{I} \subset \mathcal{K}_{N}$ provided we use an even function $f$ satisfying $f(|T|) \in \mathcal{L}^{1}$ for all $T \in \mathcal{I}$. The formula then becomes:

$$
\operatorname{Index}\left(p \mathcal{D}^{+} p\right)=(1 / f(0)) \tau\left(\gamma p f\left(\left(\left(p+(p \mathcal{D} p)^{2}\right)^{-1 / 2}\right)\right) .\right.
$$

In particular, if $(\mathcal{A}, \mathcal{H}, \mathcal{D})$ is $\theta$-summable (i.e. $e^{-t \mathcal{D}^{2}}$ is trace class for all $t>0$ ), and $f(x)=e^{-t x^{2}}, t>0$, the formula becomes:

$$
\operatorname{Index}\left(p \mathcal{D}^{+} p\right)=\tau\left(\gamma p e^{-t(p \mathcal{D} p)^{2}}\right) .
$$

\section{Semifinite spectral triples And KK-Theory}

Spectral triples in the usual $\mathcal{B}(\mathcal{H})$ case define elements of $K$-homology [23]. The consequence of [24] is that cyclic cohomology of a pre- $C^{*}$-algebra $\mathcal{A}$ entails studying semifinite Fredholm modules, in the sense of Section 8. Thus we study in this Section semifinite Kasparov modules.

In [42] semifinite spectral triples were constructed for a wide class of graph $C^{*}$-algebras. The construction of these spectral triples depended heavily on 
first constructing a Kasparov module. In [43] this relationship between Kasparov modules and semifinite spectral triples was seen again for $k$-graph algebras. In both [42] and [43] a natural compatibility was observed between the $K$-theory-valued index coming from the Kasparov module and the numerical index coming from the semifinite spectral triple. This suggests the study of the relationship between semifinite spectral triples and $K K$-theory. The results of this study are in [37] where one sees that there is a more general framework which emerges naturally. This framework is captured in the following definition.

Definition 5.1. A von Neumann spectral triple $(\mathcal{A}, \mathcal{H}, \mathcal{D})$ relative to to a pair $(\mathcal{N}, \mathcal{J})$ consists of a representation of the $*$-algebra $\mathcal{A}$ in a von Neumann algebra $\mathcal{N}$ acting on the Hilbert space $\mathcal{H}$, together with a norm closed ideal $\mathcal{J}$ and a self-adjoint operator $\mathcal{D}$ affiliated to $\mathcal{N}$ such that

(1) $[\mathcal{D}, a]$ is defined on $\operatorname{Dom}(\mathcal{D})$ and extends to a bounded operator on $\mathcal{H}$ for all $a \in \mathcal{A}$.

(2) $a(\lambda-\mathcal{D})^{-1} \in \mathcal{J}$ for all $\lambda \notin \mathbb{R}$ and $a \in \mathcal{A}$.

The spectral triple is said to be unital if the unit of $\mathcal{N}$ is in $\mathcal{A}$.

We will only consider the unital case of this definition. Recall that we write $F_{\mathcal{D}}=\mathcal{D}\left(1+\mathcal{D}^{2}\right)^{-1 / 2}$ and we let $A$ denote the $C^{*}$-closure of $\mathcal{A}$ which we assume to be separable.

The instance of this definition of greatest relevance for us is when $\mathcal{J}$ is given by $\mathcal{K}(\mathcal{N}, \tau)$, the norm closed ideal of $\mathcal{N}$ generated by the finite trace projections.

The first major result of [37] can be summarised as: from any ungraded von Neumann spectral triple $(\mathcal{A}, \mathcal{H}, \mathcal{D})$ relative to $(\mathcal{N}, \mathcal{J})$, we can construct a Kasparov module $\left(\mathcal{M}_{A}, F_{\mathcal{D}}\right)$ representing an element in $K K^{1}(A, \mathcal{J})$ where the homomorphism $\mathcal{M}_{A}: A \rightarrow \operatorname{End}_{\mathcal{J}}(\mathcal{J})$ is left multiplication by elements in $A$, and $\operatorname{End}_{\mathcal{J}}(\mathcal{J})$ is the bounded adjointable endomorphisms of the right $\mathcal{J}$ $C^{*}$-module $\mathcal{J}$. Similarly, graded spectral triples give even $K K$-classes.

The second major result rests on interpreting spectral flow in terms of the Kasparov product. First, for any unitary $u \in \mathcal{A}$, the unbounded spectral flow from $\mathcal{D}$ to $u^{*} \mathcal{D} u$ along $\left\{\mathcal{D}_{t}=(1-t) \mathcal{D}+t u \mathcal{D} u^{*}\right\}$ is defined to be the "von Neumann spectral flow' along the bounded path $\left\{F_{\mathcal{D}_{t}}=\mathcal{D}_{t}\left(\left(1+\mathcal{D}_{t}^{2}\right)^{-1 / 2}\right\}\right.$. This von Neumann spectral flow is defined in [37] to be an element of $K_{0}(\mathcal{J})$ using ideas underlying the definition of numerical spectral flow, Definition 3.1, but now regarding the index of the Fredholm pairs $\left(P_{i-1}, P_{i}\right)$ as an element of $K_{0}(\mathcal{J})$. Then it is shown in [37], assuming that $\mathcal{J}$ is $\sigma$-unital, that the unbounded spectral flow along $\left\{\mathcal{D}_{t}\right\}$ is given by the Kasparov product $[u] \otimes_{A}$ $\left[\left(\mathcal{M}_{A}, F_{\mathcal{D}}\right)\right]$ of the $K$-theory class of $u$ with the $K K$-class of $\left(\mathcal{M}_{A}, F_{\mathcal{D}}\right)$.

As $\mathcal{J}$ is typically not $\sigma$-unital, and to connect with the numerical spectral flow of previous sections, we require the following result. 
Proposition 5.2. Let $(\mathcal{A}, \mathcal{H}, \mathcal{D})$ be a von Neumann spectral triple relative to $(\mathcal{N}, \mathcal{J})$ with $\mathcal{A}$ separable in norm. Let $\mathcal{B}$ be the smallest $C^{*}$-algebra in $\mathcal{B}(\mathcal{H})$ containing the elements

$$
\begin{aligned}
& F_{\mathcal{D}}\left[F_{\mathcal{D}}, a\right] b\left[F_{\mathcal{D}}, a\right] \\
& F_{\mathcal{D}} b\left[F_{\mathcal{D}}, a\right] \quad a \varphi(\mathcal{D})
\end{aligned}
$$

for all $a, b \in \mathcal{A}$ and $\varphi \in C_{0}(\mathbb{R})$. Then $\mathcal{B}$ is separable, contained in $\mathcal{J}$ and the pair $\left(\mathcal{M}_{A}, F_{\mathcal{D}}\right)$ is a Kasparov $A$-B-module. In particular $\mathcal{B}$ is $\sigma$-unital.

Specialising to the semifinite case we have the compatibility of the Kasparov product and the numerical index given by $\tau$. The proof of this result can be found in [37].

Proposition 5.3. Let $(\mathcal{A}, \mathcal{H}, \mathcal{D}, \mathcal{N}, \tau)$ be a semifinite spectral triple such that $\left(1+\mathcal{D}^{2}\right)^{-1 / 2} \in \mathcal{L}^{p+1}(\mathcal{N}, \tau)$ and $\left[\left(1+\mathcal{D}^{2}\right)^{1 / 2}, a\right]$ is bounded for all a $\in \mathcal{A}$. Suppose also that $\mathcal{A}$ is norm separable. Let $\left[\left(\mathcal{M}_{A}, F_{\mathcal{D}}\right)\right] \in K K^{j}(A, \mathcal{B})$ be the Kasparov module associated to $(\mathcal{A}, \mathcal{H}, \mathcal{D}, \mathcal{N}, \tau)(j=0$ if the spectral triple is $\mathbb{Z}_{2}$-graded and $j=1$ otherwise). Then for $x \in K_{j}(A)$ we have

$$
\left(\tau_{*} \circ i_{*}\right)\left(x \otimes_{A}\left[\left(\mathcal{M}_{A}, F_{\mathcal{D}}\right)\right]\right)=\langle x,(\mathcal{A}, \mathcal{H}, \mathcal{D}, \mathcal{N}, \tau)\rangle
$$

where $i_{*}: K_{*}(\mathcal{B}) \rightarrow K_{*}\left(\mathcal{K}_{\mathcal{N}}\right)$ is the map induced by inclusion, $\tau_{*}: K_{0}\left(\mathcal{K}_{\mathcal{N}}\right) \rightarrow$ $\mathbb{R}$ is the map induced by the trace and the pairing on the right hand side is the analytic index pairing.

Thus we now have a clear picture of the $K$-theoretic interpretation of semifinite spectral triples and their index pairing with $K$-theory: semifinite spectral triples are specific types of representatives of $K K$ classes. This of course specialises to the original $\mathcal{B}(\mathcal{H})$ setting.

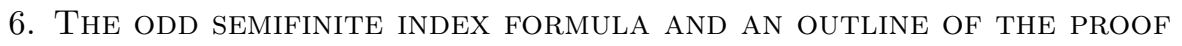

6.1. Statement of the Main Result. The statement of the theorem requires a few preliminaries. First, we require multi-indices $\left(k_{1}, \ldots, k_{m}\right), k_{i} \in$ $\{0,1,2, \ldots\}$, whose length $m$ will always be clear from the context. We write $|k|=k_{1}+\cdots+k_{m}$, and define $\alpha(k)$ by

$$
\alpha(k)^{-1}=k_{1} ! k_{2} ! \cdots k_{m} !\left(k_{1}+1\right)\left(k_{1}+k_{2}+2\right) \cdots(|k|+m) .
$$

The numbers $\sigma_{n, j}$ are defined by the equality

$$
\prod_{j=0}^{n-1}(z+j+1 / 2)=\sum_{j=0}^{n} z^{j} \sigma_{n, j} .
$$

These are just the elementary symmetric functions of $1 / 2,3 / 2, \ldots, n-1 / 2$.

Now we define, for $(\mathcal{A}, \mathcal{H}, \mathcal{D})$ having isolated spectral dimension and

$$
\begin{gathered}
b=a_{0}\left[\mathcal{D}, a_{1}\right]^{\left(k_{1}\right)} \cdots\left[\mathcal{D}, a_{m}\right]^{\left(k_{m}\right)}\left(1+\mathcal{D}^{2}\right)^{-m / 2-|k|} \\
\tau_{j}(b)=\operatorname{res}_{z=(1-p) / 2}(z-(1-p) / 2)^{j} \zeta_{b}(z-(1-q) / 2) .
\end{gathered}
$$


The hypothesis of isolated spectral dimension is clearly necessary here in order to define the residues.

With these preliminaries we can state the main result.

Theorem 6.1 (Semifinite Odd Local Index formula). Let $(\mathcal{A}, \mathcal{H}, \mathcal{D})$ be an odd finitely summable $Q C^{\infty}$ spectral triple with spectral dimension $q \geq 1$. Let $N=[q / 2]+1$ where [.] denotes the integer part, and let $u \in \mathcal{A}$ be unitary. Then

$$
s f\left(\mathcal{D}, u^{*} \mathcal{D} u\right)=\frac{1}{\sqrt{2 \pi i}} \operatorname{res}_{r=(1-q) / 2}\left(\sum_{m=1, o d d}^{2 N-1} \phi_{m}^{r}\left(C h_{m}(u)\right)\right)
$$

where for $a_{0}, \ldots, a_{m} \in \mathcal{A}, l=\{a+i v: v \in \mathbb{R}\}, 0<a<1 / 2, R_{s}(\lambda)=$ $\left(\lambda-\left(1+s^{2}+\mathcal{D}^{2}\right)\right)^{-1}$ and $r>0$ we define $\phi_{m}^{r}\left(a_{0}, a_{1}, \ldots, a_{m}\right)$ to be

$$
\frac{-2 \sqrt{2 \pi i}}{\Gamma((m+1) / 2)} \int_{0}^{\infty} s^{m} \tau\left(\frac{1}{2 \pi i} \int_{l} \lambda^{-q / 2-r} a_{0} R_{s}(\lambda)\left[\mathcal{D}, a_{1}\right] R_{s}(\lambda) \cdots\left[\mathcal{D}, a_{m}\right] R_{s}(\lambda) d \lambda\right) d s .
$$

In particular the sum on the right hand side of 1) analytically continues to a deleted neighbourhood of $r=(1-q) / 2$ with at worst a simple pole at $r=$ $(1-q) / 2$. Moreover, the complex function-valued cochain $\left(\phi_{m}^{r}\right)_{m=1, \text { odd }}^{2 N-1}$ is a $(b, B)$ cocycle for $\mathcal{A}$ modulo functions holomorphic in a half-plane containing $r=(1-q) / 2$. tions:

2) The spectral flow $s f\left(\mathcal{D}, u^{*} \mathcal{D} u\right)$ is also the residue of a sum of zeta func-

$$
\begin{aligned}
& \frac{1}{\sqrt{2 \pi i}} \operatorname{res}_{r=(1-q) / 2}\left(\sum_{m=1, o d d}^{2 N-1} \sum_{|k|=0}^{2 N-1-m|k|+(m-1) / 2} \sum_{j=0}^{2}(-1)^{|k|+m} \alpha(k) \Gamma((m+1) / 2) \sigma_{|k|+(m-1) / 2, j}\right. \\
& \left.\quad(r-(1-p) / 2)^{j} \tau\left(u^{*}[\mathcal{D}, u]^{\left(k_{1}\right)}\left[\mathcal{D}, u^{*}\right]^{\left(k_{2}\right)} \cdots[\mathcal{D}, u]^{\left(k_{m}\right)}\left(1+\mathcal{D}^{2}\right)^{-m / 2-|k|-r+(1-q) / 2}\right)\right) .
\end{aligned}
$$

In particular the sum of zeta functions on the right hand side analytically continues to a deleted neighbourhood of $r=(1-q) / 2$ and has at worst $a$ simple pole at $r=(1-q) / 2$.

3) If $(\mathcal{A}, \mathcal{H}, \mathcal{D})$ also has isolated spectral dimension then

$$
s f\left(\mathcal{D}, u^{*} \mathcal{D} u\right)=\frac{1}{\sqrt{2 \pi i}} \sum_{m} \phi_{m}\left(C h_{m}(u)\right)
$$

where for $a_{0}, \ldots, a_{m} \in \mathcal{A}$

$$
\begin{aligned}
& \phi_{m}\left(a_{0}, \ldots, a_{m}\right)=\operatorname{res}_{r=(1-p) / 2} \phi_{m}^{r}\left(a_{0}, \ldots, a_{m}\right)=\sqrt{2 \pi i} \sum_{|k|=0}^{2 N-1-m}(-1)^{|k|} \alpha(k) \\
& \quad \times \sum_{j=0}^{|k|+(m-1) / 2} \sigma_{(|k|+(m-1) / 2), j} \tau_{j}\left(a_{0}\left[\mathcal{D}, a_{1}\right]^{\left(k_{1}\right)} \ldots\left[\mathcal{D}, a_{m}\right]^{\left(k_{m}\right)}\left(1+\mathcal{D}^{2}\right)^{-|k|-m / 2}\right),
\end{aligned}
$$

and $\left(\phi_{m}\right)_{m=1, \text { odd }}^{2 N-1}$ is a $(b, B)$ cocycle for $\mathcal{A}$. When $[q]=2 n$ is even, the term with $m=2 N-1$ is zero, and for $m=1,3, \ldots, 2 N-3$, all the top terms with $|k|=2 N-1-m$ are zero. 
Remark. Since $\phi_{m}$ is a multilinear functional, it is well-defined on elements of $\mathcal{A}^{\otimes m+1}$ such as $C h_{m}(u)$.

Corollary 6.2. For $1 \leq q<2$, the statements in 3$)$ of Theorem 6.1 are true without the assumption of isolated dimension spectrum.

\subsection{Outline of the proof of the odd semifinite local index formula.} The proof may be divided initially into two parts.

(i) First we manipulate the integrand of the spectral flow formula into a form that allows us to use the resolvent expansion to obtain a perturbative formula. The individual terms in this resulting formula suggest the definition of a substitute, for finitely summable spectral triples, of the JLO cocycle of entire cyclic cohomology. Our substitute we term the 'resolvent cocycle'. It is a function-valued $(b, B)$-cocycle, modulo functions holomorphic in a certain half-plane.

(ii) The pseudodifferential calculus of [25] then enables us to write the spectral flow as a sum of zeta functions, modulo functions holomorphic in a certain half-plane. If we impose the isolated spectral dimension assumption we can analytically continue these zeta functions and take residues at a predetermined critical point. We then see that spectral flow is obtained by pairing $C h_{*}(u)$ with a variant of the Connes-Moscovici residue cocycle.

We now expand on these two basic parts. To successfully apply a perturbation technique to the spectral flow formula, Equation (2), we require 'more room to manoeuvre'. Three basic steps are involved in this part. First, using an idea of [33], we 'double-up' the data $(\mathcal{H}, \mathcal{D})$ from our spectral triple and unitary $u$ by tensoring on two copies of $\mathbb{C}^{2}$ to $\mathcal{H}$. This may be viewed as employing a formal (Clifford) Bott periodicity and replaces the trace $\tau$ by a supertrace $S \tau$.

Definition 6.3. From the Hilbert space $\tilde{\mathcal{H}}=\mathbb{C}^{2} \otimes \mathbb{C}^{2} \otimes \mathcal{H}$ acted on by the von Neumann algebra, $\tilde{\mathcal{N}}=M_{2} \otimes M_{2} \otimes \mathcal{N}$. Introduce the two dimensional Clifford algebra in the form

$$
\sigma_{1}=\left(\begin{array}{ll}
0 & 1 \\
1 & 0
\end{array}\right), \quad \sigma_{2}=\left(\begin{array}{cc}
0 & -i \\
i & 0
\end{array}\right), \quad \sigma_{3}=\left(\begin{array}{cc}
1 & 0 \\
0 & -1
\end{array}\right) .
$$

Define the grading on $\tilde{\mathcal{H}}$ by $\Gamma=\sigma_{2} \otimes \sigma_{3} \otimes 1 \in \tilde{\mathcal{N}}$.

Let $u \in \mathcal{A}$ be unitary and introduce the following even operators (i.e., they commute with $\Gamma$ ):

$$
\tilde{\mathcal{D}}=\sigma_{2} \otimes 1_{2} \otimes \mathcal{D}, \quad v=\sigma_{3} \otimes\left(\begin{array}{cc}
0 & -i u^{-1} \\
i u & 0
\end{array}\right), \quad \mathcal{D}_{r}=(1-r) \tilde{\mathcal{D}}-r v \tilde{\mathcal{D}} v
$$

The second step is to use another idea of [33] and introduce the two parameter family of operators:

$$
\mathcal{D}_{r, s}=\mathcal{D}_{r}+s v
$$


for $r \in[0,1], s \in[0, \infty)$. Clearly, the unbounded operators above are affiliated with $\tilde{\mathcal{N}}$. Notice that

$$
\mathcal{D}_{r} \equiv \mathcal{D}_{r, 0}=\sigma_{2} \otimes\left(\begin{array}{cc}
\mathcal{D}+r u^{-1}[\mathcal{D}, u] & 0 \\
0 & \mathcal{D}+r u\left[\mathcal{D}, u^{-1}\right]
\end{array}\right) .
$$

It is the appearance of both $u$ and $u^{-1}$ in this expression that requires our observation that $C h_{*}\left(u^{-1}\right)+C h_{*}(u)$ is a coboundary in the $(b, B)$ bicomplex, Equation (4).

The third step is to observe that, as the spectral flow is computed by integrating an exact one-form on an affine space of perturbations of $\tilde{\mathcal{D}}$, we may compute spectral flow from $\mathcal{D}$ to $u^{*} \mathcal{D} u$ along different paths joining the endpoints; initially it is given by integrating with respect to $r$ when $s=0$ that is along $\left\{\mathcal{D}_{r}\right\}$ and then along $\left\{\mathcal{D}_{0, s}\right\}$. The key issue here is that the spectral flow formula gives an an integral over the $s$ variable with $r=0$ in which the perturbation in (2), instead of being of first order in $\tilde{\mathcal{D}}$, is now zeroth order. Thus we obtain a new formula for spectral flow

$$
s f\left(\mathcal{D}, u^{*} \mathcal{D} u\right)=\frac{1}{C_{q / 2+r}} \int_{0}^{\infty} S \tau\left(v\left(1+\tilde{\mathcal{D}}^{2}+s\{\tilde{\mathcal{D}}, v\}+s^{2}\right)^{-q / 2-r}\right) d s
$$

where $\{\cdot, \cdot\}$ denotes the anticommutator. Crucially, the anticommutator $\{\tilde{\mathcal{D}}, v\}$ is bounded, and we are now in a position to employ perturbation theory in the form of the resolvent expansion.

Amplifying now on the second part, the pseudodifferential calculus of [25], and the 'Taylor expansion' in the form introduced by Higson, [35] are needed in order to simplify the last displayed formula. First we write

$$
\left(1+\tilde{\mathcal{D}}^{2}+s^{2}+s\{\tilde{\mathcal{D}}, v\}\right)^{-q / 2-r}=\frac{1}{2 \pi i} \int_{l} \lambda^{-q / 2-r}\left(\lambda-\left(1+\tilde{\mathcal{D}}^{2}+s^{2}+s\{\tilde{\mathcal{D}}, v\}\right)\right)^{-1} d \lambda
$$

where the vertical line $l$ lies between 0 and $\operatorname{spec}\left(1+\tilde{\mathcal{D}}^{2}+s^{2}+s\{\tilde{\mathcal{D}}, v\}\right)$ for all $s \in[0, \infty)$. We then apply the resolvent expansion (writing $R_{s}(\lambda)=$ $\left.\left(\lambda-\left(1+\tilde{\mathcal{D}}^{2}+s^{2}\right)\right)^{-1}\right)$

$\left(\lambda-\left(1+\tilde{\mathcal{D}}^{2}+s^{2}+s\{\tilde{\mathcal{D}}, v\}\right)\right)^{-1}=\sum_{m=0}^{2 N-1}\left(R_{s}(\lambda) s\{\tilde{\mathcal{D}}, v\}\right)^{m} R_{s}(\lambda)+$ Remainder.

Then modulo functions of $r$ holomorphic in a half-plane containing $r=(1-$ q) $/ 2$

$$
s f\left(\mathcal{D}, u^{*} \mathcal{D} u\right) C_{q / 2+r}=\frac{1}{2 \pi i} \sum_{m=1, o d d}^{2 N-1} \int_{0}^{\infty} s^{m} S \tau\left(p \int_{l} \lambda^{-q / 2-r}\left(R_{s}(\lambda)\{\tilde{\mathcal{D}}, v\}\right)^{m} R_{s}(\lambda) d \lambda\right) d s .
$$

The even terms in the expansion (6) are seen to vanish by elementary Cliffordtype manipulations. The 'constant'

$$
C_{q / 2+r}=\frac{\Gamma(r-(1-q) / 2) \Gamma(1 / 2)}{\Gamma(q / 2+r)}
$$


has simple poles at $r=(1-q) / 2-k, k=0,1,2, \ldots$, with residue equal to 1 at $r=(1-q) / 2$. Therefore, since the error terms in Equation (7) are holomorphic at $r=(1-q) / 2$, we may take residues at $r=(1-q) / 2$ of the analytic continuations of both sides of (7) even though the individual terms in this expansion need not analytically continue.

One may perform the 'super' part of the trace to obtain a formula for the spectral flow in terms of the original spectral triple $(\mathcal{A}, \mathcal{H}, \mathcal{D})$ and the unitary $u$. The general structure of this formula suggests the definition of a function-valued $(b, B)$-cochain on the algebra $\mathcal{A}$, the resolvent cocycle. Using techniques inspired by Higson, [35], we show that this is a cocycle modulo functions of $r$ holomorphic in a half-plane containing $(1-q) / 2$. This 'almost cocycle' property proves 1 ) of our theorem above.

Returning to our spectral flow computations we apply the pseudodifferential calculus to each term of the resolvent expansion. This moves all the resolvents to the right, allowing us to use Cauchy's formula to perform the complex line integral. We then perform the remaining integral over $s \in[0, \infty)$, and so obtain our penultimate formula:

$$
\begin{aligned}
& s f\left(\mathcal{D}, u^{*} \mathcal{D} u\right) C_{q / 2+r} \\
& =\sum_{m=1, \text { odd }}^{2 N-1} \sum_{|k|=0}^{2 N-1-m} C_{k, m, r} S \tau\left(v\{\tilde{\mathcal{D}}, v\}^{\left(k_{1}\right)} \cdots\{\tilde{\mathcal{D}}, v\}^{\left(k_{m}\right)}\left(1+\tilde{\mathcal{D}}^{2}\right)^{-(q-1) / 2-|k|-m / 2-r}\right),
\end{aligned}
$$

where equality is again modulo functions of $r$ holomorphic in a half-plane containing $(1-q) / 2$. That such a formula exists is surprising (and for $q<2$ quite useful) as we have not invoked the isolated spectral dimension hypothesis. The sum of zeta functions in Equation (8) clearly has a simple pole at $r=(1-q) / 2$, with residue equal to the spectral flow. This proves part 2$)$ of our theorem.

Finally assume that the individual zeta functions possess analytic continuations to a deleted neighbourhod of $r=(1-q) / 2$ so we can take residues of the zeta functions to obtain our version of the residue cocycle. We can then prove part 3) of the theorem. The cocycle property for the residue cocycle follows from the 'almost' cocycle property of the resolvent cocycle upon taking residues.

6.3. Some novel aspects of our approach. We emphasise that there are important differences in our approach from that of [25] and [35].

- Only the final step of both of our proofs requires an analytic continuation property of the generalised zeta functions that are derived from the resolvent cocycle. Indeed, we express spectral flow and the index in both the odd and even (see Section 7) cases as the residue of a sum of zeta functions without invoking any analytic continuation hypothesis. Naturally our results specialise to the standard $\mathcal{B}(\mathcal{H})$ case. 
- We make no assumptions on the decay of our zeta functions along vertical lines in the complex plane thus reducing the side conditions that need to be checked when applying the local index formula of [25].

- Our proof that the residue cocycle [25] is indeed a $(b, B)$-cocycle is quite simple even in the general semifinite case by virtue of using the algebraic properties of the resolvent cocycle.

- The strategy of both proofs is straightforward and rests on algebraic calculations. Of course the algebra has to be justified at various points using some sharp estimates (but these all have elementary proofs).

- We remark that there is an unrenormalised version of the residue cocycle in [25] containing an infinite number of terms in the case that one of the terms in the expansion has an essential singularity, whereas the renormalised version in [25] always has a bounded number of terms. The unrenormalised version presents an issue of convergence which is difficult to address. Since we do not pass through an intermediate step where the cocycle contains a potentially infinite number of terms, we are free to allow essential singularities from the outset.

\section{The EVEN CASE OF THE LOCAL INDEX FORMUlA}

We write $P=(1+\gamma) / 2$ and $\mathcal{D}^{+}=(1-P) \mathcal{D} P=P^{\perp} \mathcal{D} P$. The operator $\mathcal{D}^{+}: \mathcal{H}^{+}=P(\mathcal{H}) \rightarrow \mathcal{H}^{-}=P^{\perp}(\mathcal{H})$ is, as we shall see, an unbounded BreuerFredholm operator.

As we remarked previously the articles $[23,40,16]$ contain the details of the definition of the $(b, B)$ bicomplex. The $(b, B)$ Chern character of a projection in an algebra $\mathcal{A}$ is an even $(b, B)$ cycle with $2 m$-th term, $m \geq 1$, given by

$$
C h_{2 m}(p)=(-1)^{m} \frac{(2 m) !}{2(m !)}(2 p-1) \otimes p^{\otimes 2 m} .
$$

For $m=0$ the definition is $C h_{0}(p)=p$.

For our main result in the even case we use the same notation as in the odd case, with the exception that $\sigma_{n, j}$ shall denote the elementary symmetric functions of $\{1, \ldots, n\}$, i.e.

$$
\prod_{j=0}^{n-1}(z+j)=\sum_{j=1}^{n} z^{j} \sigma_{n, j}
$$

We recall the notation $q=\inf \left\{k \in \mathbf{R}: \tau\left(\left(1+\mathcal{D}^{2}\right)^{-k / 2}\right)<\infty\right\}$ for the spectral dimension of $(\mathcal{A}, \mathcal{H}, \mathcal{D})$ and we assume it is isolated so that $\tau_{j}(b)=$ res $_{z=(1-q) / 2}(z-(1-q) / 2)^{j} \zeta_{b}(z-(1-q) / 2)$ is defined.

Theorem 7.1 (Semifinite Even Local Index formula). Let $(\mathcal{A}, \mathcal{H}, \mathcal{D})$ be an even $Q C^{\infty}$ spectral triple with spectral dimension $q \geq 1$. Let $N=\left[\frac{q+1}{2}\right]$, where $[\cdot]$ denotes the integer part, and let $p \in \mathcal{A}$ be a self-adjoint projection. Then 
1) $\quad \operatorname{Index}\left(p \mathcal{D}^{+} p\right)=r e s_{r=(1-q) / 2}\left(\sum_{m=0, \text { even }}^{2 N} \phi_{m}^{r}\left(C h_{m}(p)\right)\right)$ where for $a_{0}, \ldots, a_{m} \in \mathcal{A}, l=\{a+i v: v \in \mathbb{R}\}, 0<a<1 / 2, R_{s}(\lambda)=$ $\left(\lambda-\left(1+s^{2}+\mathcal{D}^{2}\right)\right)^{-1}$ and $r>1 / 2$ we define $\phi_{m}^{r}\left(a_{0}, a_{1}, \ldots, a_{m}\right)$ to be

$$
\frac{(m / 2) !}{m !} \int_{0}^{\infty} 2^{m+1} s^{m} \tau\left(\gamma \frac{1}{2 \pi i} \int_{l} \lambda^{-q / 2-r} a_{0} R_{s}(\lambda)\left[\mathcal{D}, a_{1}\right] R_{s}(\lambda) \cdots\left[\mathcal{D}, a_{m}\right] R_{s}(\lambda) d \lambda\right) d s .
$$

In particular the sum on the right hand side of 1) analytically continues to a deleted neighbourhood of $r=(1-q) / 2$ with at worst a simple pole at $r=$ $(1-q) / 2$. Moreover, the complex function-valued cochain $\left(\phi_{m}^{r}\right)_{m=0, \text { even }}^{2 N}$ is a $(b, B)$ cocycle for $\mathcal{A}$ modulo functions holomorphic in a half-plane containing $r=(1-q) / 2$.

2) The index, $\operatorname{Index}\left(p \mathcal{D}^{+} p\right)$ is also the residue of a sum of zeta functions:

$$
\begin{aligned}
& \operatorname{res}_{r=(1-q) / 2}\left(\sum_{m=0, \text { even }}^{2 N} \sum_{|k|=0}^{2 N-m|k|+m / 2} \sum_{j=1}^{\mid}(-1)^{|k|+m / 2} \alpha(k) \frac{(m / 2) !}{2 m !} \sigma_{|k|+m / 2, j}\right. \\
& \left.\times(r-(1-q) / 2)^{j} \tau\left(\gamma(2 p-1)[\mathcal{D}, p]^{\left(k_{1}\right)}[\mathcal{D}, p]^{\left(k_{2}\right)} \cdots[\mathcal{D}, p]^{\left(k_{m}\right)}\left(1+\mathcal{D}^{2}\right)^{-m / 2-|k|-r+(1-q) / 2}\right)\right),
\end{aligned}
$$

(for $m=0$ we replace $(2 p-1)$ by $2 p)$. In particular the sum of zeta functions on the right hand side analytically continues to a deleted neighbourhood of $r=(1-q) / 2$ and has at worst a simple pole at $r=(1-q) / 2$.

3) If $(\mathcal{A}, \mathcal{H}, \mathcal{D})$ also has isolated spectral dimension then

$$
\operatorname{Index}\left(p \mathcal{D}^{+} p\right)=\sum_{m=0, \text { even }}^{2 N} \phi_{m}\left(C h_{m}(p)\right)
$$

where for $a_{0}, \ldots, a_{m} \in \mathcal{A}$ we have $\phi_{0}\left(a_{0}\right)=\operatorname{res}_{r=(1-q) / 2} \phi_{0}^{r}\left(a_{0}\right)=\tau_{-1}\left(\gamma a_{0}\right)$ and for $m \geq 2$

$$
\begin{aligned}
\phi_{m}\left(a_{0}, \ldots, a_{m}\right) & =r e s_{r=(1-q) / 2} \phi_{m}^{r}\left(a_{0}, \ldots, a_{m}\right)=\sum_{|k|=0}^{2 N-m}(-1)^{|k|} \alpha(k) \\
& \times \sum_{j=1}^{|k|+m / 2} \sigma_{(|k|+m / 2), j} \tau_{j-1}\left(\gamma a_{0}\left[\mathcal{D}, a_{1}\right]^{\left(k_{1}\right)} \ldots\left[\mathcal{D}, a_{m}\right]^{\left(k_{m}\right)}\left(1+\mathcal{D}^{2}\right)^{-|k|-m / 2}\right),
\end{aligned}
$$

and $\left(\phi_{m}\right)_{m=0, \text { even }}^{2 N}$ is a $(b, B)$ cocycle for $\mathcal{A}$. When $[q]=2 n+1$ is odd, the term with $m=2 N$ is zero, and for $m=0,2, \ldots, 2 N-2$, all the top terms with $|k|=2 N-m$ are zero.

Corollary 7.2. For $1 \leq q<2$, the statements in 3$)$ of our theorem are true without the assumption of isolated dimension spectrum.

The proof of this result is very similar to the odd case if one starts from the generalised McKean-Singer formula instead of the spectral flow formula. We will not repeat the overview here, see [17]. 
8. The homotopy to the Chern CharaCter in SEmifinite NCG.

As we explained in the introduction, there is another way to prove the local index formula in the semifinite situation. The idea is to show that the resolvent cocycle is an index cocycle by a direct argument using a homotopy to the semifinite Chern character. To define the Chern character of a finitely summable Fredholm module in this generality we start with a definition.

Definition 8.1. A pre-Fredholm module for a unital topological $*$-algebra $\mathcal{A}$ is a pair $(\mathcal{H}, F)$ where $\mathcal{A}$ is (continuously) represented in $\mathcal{N}$ (a semifinite von Neumann algebra acting on $\mathcal{H})$ and $F$ is a self-adjoint Breuer-Fredholm operator in $\mathcal{N}$ satisfying:

$1.1-F^{2} \in \mathcal{K}_{\mathcal{N}}$, and

2. $[F, a] \in \mathcal{K}_{\mathcal{N}}$ for $a \in \mathcal{A}$.

If $1-F^{2}=0$ we drop the prefix "pre-". If $[F, a] \in \mathcal{L}^{n+1}(\mathcal{N})$ for all $a \in \mathcal{A}$, we say that $(\mathcal{H}, F)$ is $n+1$-summable.

If there is a self adjoint involution $\Gamma$ with $\Gamma F+F \Gamma=0$ and $\Gamma a-a \Gamma=0$ for all $a \in \mathcal{A}$, then we say the pre-Fredholm module is even, otherwise it is odd.

Semifinite spectral triples give rise to pre-Fredholm modules via

$$
(\mathcal{A}, \mathcal{H}, \mathcal{D}) \longrightarrow\left(\mathcal{H}, F=\mathcal{D}\left(1+\mathcal{D}^{2}\right)^{-1 / 2}\right) .
$$

One views spectral triples as geometric representatives of $K$-homology classes, in much the same way that one views differential forms.

If the semifinite spectral triple $(\mathcal{A}, \mathcal{H}, \mathcal{D})$ is $Q C^{\infty}$ and finitely summable with $\left(1+\mathcal{D}^{2}\right)^{-s / 2}$ trace class for all $s>q$, and has $\mathcal{D}$ invertible, then

$$
\left(\mathcal{H}, F=\mathcal{D}|\mathcal{D}|^{-1}\right)
$$

is a $[q]+1$-summable Fredholm module where $[\cdot]$ denotes the integer part.

Given a Fredholm module $(\mathcal{H}, F)$ define the 'conditional trace' $\tau^{\prime}$ by

$$
\tau^{\prime}(T)=\frac{1}{2} \tau(F(F T+T F))
$$

provided $F T+T F \in \mathcal{L}^{1}(\mathcal{N})$ (as it will be in our case, see [23, p293]). Note that if $T \in \mathcal{L}^{1}(\mathcal{N})$ we have (using the trace property and $F^{2}=1$ )

$$
\tau^{\prime}(T)=\tau(T)
$$

The Chern character $\left[C h_{F}\right]$ of an $n+1$-summable Fredholm module $(\mathcal{H}, F)$ is the class in periodic cyclic cohomology of the cyclic cocycles (setting $\Gamma=1$ for an odd Fredholm module)

$\lambda_{m} \tau^{\prime}\left(\Gamma a_{0}\left[F, a_{1}\right] \cdots\left[F, a_{m}\right]\right), \quad a_{0}, \ldots, a_{n} \in \mathcal{A}, \quad m \geq n, m$ even if $(\mathcal{H}, F)$ even, and odd otherwise. 
Here $\lambda_{n}$ are constants ensuring that this collection of cocycles yields a well-defined periodic class, and they are given by

$$
\lambda_{n}=\left\{\begin{array}{lll}
(-1)^{n(n-1) / 2} \Gamma\left(\frac{n}{2}+1\right) & n & \text { even } \\
\sqrt{2 i}(-1)^{n(n-1) / 2} \Gamma\left(\frac{n}{2}+1\right) & n & \text { odd }
\end{array} .\right.
$$

The class of the Chern character of an $n+1$-summable Fredholm module is represented by the cyclic cocycle $C h_{F} \in C_{\lambda}^{n}(\mathcal{A})$

$$
C h_{F}\left(a_{0}, \ldots, a_{n}\right)=\lambda_{n} \tau^{\prime}\left(\Gamma a_{0}\left[F, a_{1}\right] \cdots\left[F, a_{n}\right]\right), \quad a_{0}, \ldots, a_{n} \in \mathcal{A} .
$$

We will always take the cyclic cochain $C h_{F}$ (or its $(b, B)$ analogue; see below) as representative of $\left[C h_{F}\right]$, and will often refer to $C h_{F}$ as the Chern character.

Since the Chern character is a cyclic cochain, it lies in the image of the operator $B$, [23, Corollary 20, III.1. $\beta]$, and so $B C h_{F}=0$ since $B^{2}=0$. As $b C h_{F}=0$, we may regard the Chern character as a one term element of the $(b, B)$ bicomplex. However, the correct normalisation is (taking the Chern character to be in degree $n$ )

$$
C_{\lambda}^{n} \ni C h_{F} \longrightarrow \frac{(-1)^{[n / 2]}}{n !} C h_{F} \in C^{n} .
$$

Thus instead of $\lambda_{n}$ defined above, we use $\mu_{n}$

$$
\mu_{n}=\frac{(-1)^{[n / 2]}}{n !} \lambda_{n}=\left\{\begin{array}{lll}
\frac{\Gamma\left(\frac{n}{2}+1\right)}{n !} & n & \text { even } \\
\sqrt{2 i} \frac{\Gamma\left(\frac{n}{2}+1\right)}{n !} & n & \text { odd }
\end{array} .\right.
$$

The difference in normalisation between periodic and $(b, B)$ is due solely to the way the index pairing is defined in the two cases, [23].

Our next task is to show that if our spectral triple $(\mathcal{A}, \mathcal{H}, \mathcal{D})$ is such that $\mathcal{D}$ is not invertible, we can replace it by a new spectral triple in the same $K K$-class in which the unbounded operator is invertible.

Definition 8.2. Let $(\mathcal{A}, \mathcal{H}, \mathcal{D})$ be a spectral triple. For any $\mu \in \mathbb{R} \backslash\{0\}$, define the 'double' of $(\mathcal{A}, \mathcal{H}, \mathcal{D})$ to be the spectral triple $\left(\mathcal{A}, \mathcal{H}^{2}, \mathcal{D}_{\mu}\right)$ with $\mathcal{H}^{2}=\mathcal{H} \oplus \mathcal{H}$, and the action of $\mathcal{A}$ and $\mathcal{D}_{\mu}$ given by

$$
\mathcal{D}_{\mu}=\left(\begin{array}{cc}
\mathcal{D} & \mu \\
\mu & -\mathcal{D}
\end{array}\right), \quad a \rightarrow\left(\begin{array}{ll}
a & 0 \\
0 & 0
\end{array}\right), \quad \forall a \in \mathcal{A} .
$$

Whether $\mathcal{D}$ is invertible or not, $\mathcal{D}_{\mu}$ always is invertible, and $F_{\mu}:=\mathcal{D}_{\mu}\left|\mathcal{D}_{\mu}\right|^{-1}$ has square 1 . This is the chief reason for introducing this construction.

The original statement in the general semifinite case, [13], was stated in terms of 'semifnite $K$-homology classes'. There we said that two (semifinite) spectral triples were in the same $K$-homology class, if the associated preFredholm modules were operator homotopic up to the addition of degenerate Fredholm modules. With the observations in Section 5 from [37], and the proof presented in [13] we may conclude that 
Lemma 8.3. The $K K$-classes of $(\mathcal{A}, \mathcal{H}, \mathcal{D})$ and $\left(\mathcal{A}, \mathcal{H}^{2}, \mathcal{D}_{\mu}\right)$ are the same. $A$ representative of this class is $\left(\mathcal{B} \oplus \mathcal{B}, F_{\mu}\right)$ with $F_{\mu}=\mathcal{D}_{\mu}\left|\mathcal{D}_{\mu}\right|^{-1}$, where $\mathcal{B} \subset \mathcal{K}_{\mathcal{N}}$ is as in Proposition 5.2.

The most basic consequence of Lemma 8.3 comes from the following (see [28, pp65-68] for the proof, which is the same in this setting).

Proposition 8.4. The periodic cyclic cohomology class of the Chern character of a finitely summable Fredholm module depends only on its K-homology class.

In particular, therefore, the Chern characters of $(\mathcal{A}, \mathcal{H}, \mathcal{D})$ and $\left(\mathcal{A}, \mathcal{H}^{2}, \mathcal{D}_{\mu}\right)$ have the same class in periodic cyclic cohomology, and this can be computed (indeed is defined!) using the Fredholm module $\left(\mathcal{H}^{2}, F_{\mu}\right)$, and this class is independent of $\mu$.

8.1. The resolvent cocycle in the general case. The following definition establishes some notation needed to treat the even and odd cases on the same footing.

Definition 8.5. Let $(\mathcal{A}, \mathcal{H}, \mathcal{D})$ be a spectral triple with spectral dimension $q \geq 1$. Let $P$ denote the parity of the triple, so $P=0$ for even triples and $P=1$ for odd triples. Let $A$ denote $(P-1) \bmod 2$, so $A=1$ for even triples and $A=0$ for odd triples. We adopt the convention that $|\mathcal{D}|$ and elements of $\mathcal{A}$ have 'grading degree' zero, while $\mathcal{D}$ has grading degree one. In the even case this is of course the actual grading degree of the spectral triple. We denote the grading degree of $T$ in the algebra generated by $\mathcal{D}$ and $\mathcal{A}$ by $\operatorname{deg}(T)$ (see $[16,25]$ for more detail on the allowed $T$ 's $)$. Finally, let $N=[(q+1+P) / 2]$ where [.] denotes the integer part.

The grading degree is used to define the graded commutator

$$
[T, R]_{ \pm}:=T R-(-1)^{\operatorname{deg}(T) \operatorname{deg}(R)} R T .
$$

The following definition generalises the expectations introduced in $[16,17]$ to deal with both the even and odd cases in a uniform fashion.

It is also useful to let the order of $T$ in the algebra generated by $\mathcal{D}$ and $\mathcal{A}$ be the number of powers of $\mathcal{D}$ in $T$. This loose definition is made precise by the pseudodifferential calculus for spectral triples described in $[16,25]$.

Definition 8.6. Let $0<a<1 / 2$ and let $l$ be the vertical line $l=\{a+i v: v \in$ $\mathbb{R}\}$. For $m \geq 0, s \in[0, \infty)$ and operators $A_{0}, \ldots, A_{m}$ in the algebra generated by $\mathcal{D}$ and $\mathcal{A}$ with the order of $A_{j}$ equal to $k_{j}$, and $k_{0}+\cdots+k_{m}-2 m<2 R e(r)$, define

$$
\left\langle A_{0}, \ldots, A_{m}\right\rangle_{m, s, r}=\tau\left(\frac{1}{2 \pi i} \Gamma \int_{l} \lambda^{-q / 2-r} A_{0} R_{s}(\lambda) A_{1} \cdots A_{m} R_{s}(\lambda) d \lambda\right) .
$$


Here $\Gamma$ is the $\mathbb{Z}_{2}$-grading in the even case and the identity operator in the odd case, and $R_{s}(\lambda)=\left(\lambda-\left(1+s^{2}+\mathcal{D}^{2}\right)\right)^{-1}$.

We now state the definition of the resolvent cocycle in terms of the expectations $\langle\cdots\rangle_{m, s, r}$.

Definition 8.7. Let $\eta_{m}$ denote the following constant

$$
\eta_{m}=(-\sqrt{2 i})^{P} 2^{m+1} \frac{\Gamma(m / 2+1)}{\Gamma(m+1)}
$$

and write da for the commutator $[\mathcal{D}, a]$. Then for $\operatorname{Re}(r)>\frac{1}{2}(1-m)$ we define the $m$-th component of the resolvent cocycle $\phi_{m}^{r}: \mathcal{A}^{\otimes m+1} \rightarrow \mathbb{C}$ by

$$
\phi_{m}^{r}\left(a_{0}, \ldots, a_{m}\right)=\eta_{m} \int_{0}^{\infty} s^{m}\left\langle a_{0}, d a_{1}, . ., d a_{m}\right\rangle_{m, s, r} d s .
$$

8.2. The residue cocycle. We use the notation above to write down the residue cocycle.

Definition 8.8. Let $(\mathcal{A}, \mathcal{H}, \mathcal{D})$ be a $Q C^{\infty}$ finitely summable spectral triple with isolated spectral dimension $q \geq 1$. Let $M=2 N-P$. For $m=P, P+2, \ldots, M$, define functionals $\phi_{m}$ by

$$
\begin{aligned}
& \phi_{m}\left(a_{0}, \ldots, a_{m}\right) \\
& =\sqrt{2 \pi i} \sum_{|k|=0}^{M-m}(-1)^{|k|} \alpha(k) \sum_{j=A}^{h} \sigma_{h, j} \tau_{j-A}\left(\Gamma a_{0}\left[\mathcal{D}, a_{1}\right]^{\left(k_{1}\right)} \ldots\left[\mathcal{D}, a_{m}\right]^{\left(k_{m}\right)}\left(1+\mathcal{D}^{2}\right)^{-|k|-m / 2}\right),
\end{aligned}
$$

where $h=|k|+(m-P) / 2$. Here $\Gamma$ denotes the $\mathbb{Z}_{2}$-grading in the even case and the identity operator in the odd case.

It follows from $[16,17]$ that $\phi=\left(\phi_{m}\right)_{m=P}^{M}$ is a $(b, B)$-cocycle, called the residue cocycle.

The relationship between the resolvent cocycle and the residue cocycle is captured by the following result $[16,17]$.

Theorem 8.9. Let $(\mathcal{A}, \mathcal{H}, \mathcal{D})$ be a $Q C^{\infty}$ finitely summable spectral triple with isolated spectral dimension $q \geq 1$. When evaluated on any $a_{0}, \ldots, a_{m} \in \mathcal{A}$, the components $\phi_{m}^{r}$ of the resolvent cocycle $\left(\phi^{r}\right)$ analytically continue to a deleted neighbourhood of $r=(1-q) / 2$. Moreover, if we denote this continuation by $\varphi_{m}^{r}\left(a_{0}, \ldots, a_{m}\right)$ then

$$
\operatorname{res}_{r=(1-q) / 2} \varphi_{m}^{r}\left(a_{0}, \ldots, a_{m}\right)=\phi_{m}\left(a_{0}, \ldots, a_{m}\right) .
$$


8.3. The Residue Cocycle Represents the Chern Character. The main fact about this situation which is proved in [18] is as follows.

Theorem 8.10. Let $(\mathcal{A}, \mathcal{H}, \mathcal{D})$ be a $Q C^{\infty}$ spectral triple (even or odd) with spectral dimension $q \geq 1$ and isolated spectral dimension. Then the residue cocycle represents the Chern character of the $K$-homology class of $(\mathcal{A}, \mathcal{H}, \mathcal{D})$.

This theorem completes the circle of ideas suggested by the approach in $[16,17]$ to the local index formula. Its proof, via the use of the resolvent cocycle, shows that the resolvent cocycle is indeed playing the same role as the JLO cocycle does for the original proof of the local index formula.

The proof of the theorem rests on two results. The first is:

Theorem 8.11. Let $(\mathcal{A}, \mathcal{H}, \mathcal{D})$ be a $Q C^{\infty}$ finitely summable spectral triple with dimension $q \geq 1$ and $\mathcal{D}$ invertible. Let $M=2 N-P$ where $N=[(q+$ $1+P) / 2]$. Then in the $(b, B)$ bicomplex for $\mathcal{A}$ with coefficients in functions holomorphic for $\operatorname{Re}(r)>1 / 2$, the resolvent cocycle $\left(\phi_{m}^{r}\right)_{m=P}^{M}$ is cohomologous to

$$
\frac{1}{(r-(1-q) / 2)} C h_{F}^{M}
$$

modulo cochains with values in the functions holomorphic in a half-plane containing $r=(1-q) / 2$. Here $F=\mathcal{D}|\mathcal{D}|^{-1}$, and $C h_{F}^{M}$ denotes the representative of the Chern character in dimension $M$.

The second fact that is needed is:

Theorem 8.12. If $(\mathcal{A}, \mathcal{H}, \mathcal{D})$ is a $Q C^{\infty}$ spectral triple with isolated spectral dimension $q \geq 1$ and $\mathcal{D}$ invertible, then the cyclic cohomology class of the residue cocycle coincides with the class of the Chern character of $(\mathcal{H}, F=$ $\left.\mathcal{D}|\mathcal{D}|^{-1}\right)$.

There are two main steps involved in proving these statements. First we need to define a 'transgression' cochain which provides an exact cohomology between the resolvent cocycle and a single term cyclic cochain which is 'almost' a cocycle. Then we must deform the resulting single term cyclic cochain to the Chern character. In this process we introduce error terms that are holomorphic at $r=(1-q) / 2$. The second theorem follows on taking residues, which requires the isolated spectral dimension hypothesis.

Both these steps require invertibility of $\mathcal{D}$. However, once we have proved the result for invertible $\mathcal{D}$, we can replace $(\mathcal{A}, \mathcal{H}, \mathcal{D})$ by its double to remove this hypothesis. 


\section{Connection With Higson's Cocycle}

Higson has a cocycle which is evidently similar to our resolvent cocycle. An essential difference is that from Higson's cocycle, one derives the unrenormalised local index theorem. We show here that our resolvent cocycle naturally gives rise to a 'renormalised' version of Higson's cocycle.

We take our resolvent cocycle, perform the pseudodifferential expansion, the Cauchy integral and the $s$-integral. This gives (modulo functions holomorphic at $r=(1-q) / 2)$

$$
\begin{aligned}
\phi_{m}^{r}\left(a_{0}, \ldots, a_{m}\right)= & \sum_{|k|=0}^{2 N-m-P} C(k)(-1)^{m+|k|} \sqrt{\pi}(-1)^{P} \sqrt{2 i}^{P} \frac{\Gamma(|k|+(m-1) / 2+q / 2+r)}{\Gamma(1+|k|+m) \Gamma(q / 2+r)} \\
& \times \tau\left(\gamma a_{0}\left[\mathcal{D}, a_{1}\right]^{\left(k_{1}\right)} \cdots\left[\mathcal{D}, a_{m}\right]^{\left(k_{m}\right)}\left(1+\mathcal{D}^{2}\right)^{-q / 2-r-|k|-(m-1) / 2}\right)
\end{aligned}
$$

We then put back the Cauchy integral using

$$
\begin{aligned}
& \tau\left(\gamma a_{0}\left[\mathcal{D}, a_{1}\right]^{\left(k_{1}\right)} \cdots\left[\mathcal{D}, a_{m}\right]^{\left(k_{m}\right)}\left(1+\mathcal{D}^{2}\right)^{-q / 2-r-|k|-(m-1) / 2}\right) \\
& (-1)^{|k|+m} \frac{\Gamma(1+|k|+m) \Gamma(q / 2+r-(m+1) / 2)}{\Gamma(q / 2+r+|k|+(m-1) / 2)} \\
& \times \tau\left(\frac{1}{2 \pi i} \int_{l} \lambda^{-q / 2-r+(m+1) / 2} a_{0}\left[\mathcal{D}, a_{1}\right]^{\left(k_{1}\right)} \cdots\left[\mathcal{D}, a_{m}\right]^{\left(k_{m}\right)}\left(\lambda-\left(1+\mathcal{D}^{2}\right)\right)^{-|k|-m-1} d \lambda\right)
\end{aligned}
$$

and undo the pseudodifferential expansion. Our estimates show that these operations affect our function-valued cocycle only by functions holomorphic at the critical point $r=(1-q) / 2$. We obtain the following equality modulo functions holomorphic at $r=(1-q) / 2$ :

$$
\begin{aligned}
& \phi_{m}^{r}\left(a_{0}, \ldots, a_{m}\right)=(-1)^{P} \sqrt{2 i}^{P} \frac{\sqrt{\pi} \Gamma(q / 2+r-(m+1) / 2)}{\Gamma(q / 2+r)} \\
& \times \tau\left(\frac{1}{2 \pi i} \int_{l} \lambda^{-q / 2-r+(m+1) / 2} a_{0} R_{0}(\lambda)\left[\mathcal{D}, a_{1}\right] R_{0}(\lambda) \cdots\left[\mathcal{D}, a_{m}\right] R_{0}(\lambda) d \lambda\right) .
\end{aligned}
$$

We call this new cocycle the reduced resolvent cocycle, and denote its components by $\psi_{m}^{r}$ so that the above equality becomes

$$
\phi_{m}^{r}\left(a_{0}, \ldots, a_{m}\right)=\psi_{m}^{r}\left(a_{0}, \ldots, a_{m}\right)
$$

modulo functions holomorphic at $r=(1-q) / 2$. The integral defining $\psi_{m}^{r}$ exists for $\operatorname{Re}(r)>(1-m) / 2$ by our previous estimates. The argument of the coefficent

$$
\Gamma(q / 2+r-(m+1) / 2)
$$

has positive real part when $\operatorname{Re}(r)>m / 2+(1-q) / 2$, and can be meromorphically continued.

To compare the reduced resolvent cocycle with Higson's improper cocycle, we write $z=r-(1-q) / 2$. Then, writing $\eta_{m}^{z}$ for the components of Higson's improper cocycle we have

$$
\psi_{m}^{r}=\frac{\sqrt{\pi}}{\Gamma(z+1 / 2)} \eta_{m}^{z} .
$$


This gives a 'renormalised' version of Higson's cocycle in the sense that starting with the reduced resolvent cocycle, one arrives at the renormalised local index theorem, whereas Higson's original cocycle leads to the unrenormalised theorem.

\section{Applications}

In an earlier review of analytic spectral flow in von Neumann algebras [2] we explained how a number of older results such as those in $[22,31,39,48,51]$ could be seen to be examples of semifinite NCG index theorems. For foliations that give rise to type II von Neumann algebras [3] explains the relevance of the semifinite theory.

Graph $C^{*}$-algebras that admit traces are shown to lead naturally to semifinite spectral triples in [42] and the local index formula may be used to obtain an index formula for generalised Toeplitz type operators. Analogous results are proved for the $C^{*}$-algebras of $k$-graphs in [43] where the spectral dimension is found to be $k$. These results are explained in a more general setting of compact group actions on $C^{*}$-algebras in [52]. Semifinite spectral triples were found to arise in an application of spectral triples to loop quantum gravity in [1].

Having established a general framework for semifinite NCG it is tempting to move on to see how this might be exploited to produce index theorems in type III von Neumann algebras. A start on such a program is given in $[7,15,20]$.

\section{REFERENCES}

[1] J. Aastrup, J. Grimstrup, R. Nest, On spectral triples in quantum gravity I, Classical Quantum Gravity 26 no. 6, (2009), 53pp.

[2] M.-T. Benameur, A. L. Carey, J. Phillips, A. Rennie, F. A. Sukochev, and K. P. Wojciechowski, An analytic approach to spectral flow in von Neumann algebras in Analysis, geometry and topology of elliptic operators, pp 297-352. World Sci. Publ., Hackensack, NJ, 2006.

[3] M.T. Benameur and T. Fack, Type II non-commutative geometry. I. Dixmier trace in von Neumann algebras, Advances in Mathematics 199 2006, 29-87.

[4] M. Breuer, Fredholm theories in von Neumann algebras. I, Math. Ann., 178(1968), $243-254$.

[5] M. Breuer, Fredholm theories in von Neumann algebras. II, Math. Ann., 180(1969), 313-325.

[6] A. Carey, M. Marcolli, A. Rennie, Modular index invariants of mumford curves, archive-math:0905-3157.

[7] A.L. Carey, S. Neshveyev, R. Nest and A. Rennie, Twisted cyclic theory, equivariant $K K$ theory and KMS States, to appear in Crelle's journal, arXiv:0808.3029.

[8] A. L. Carey, J. Phillips, Algebras almost commuting with Clifford algebras in a $I_{\infty}$ factor, K-theory 4 (1991), 445-478.

[9] A. L. Carey, J. Phillips, Unbounded Fredholm modules and spectral flow, Canadian J. Math., 50(1998), 673-718.

[10] A. L. Carey, J. Phillips, Spectral flow in $\theta$-summable Fredholm modules, eta invariants and the JLO cocycle, K-Theory, 31 (2004), 135-194. 
[11] A. L. Carey, J. Phillips, F. A. Sukochev, On unbounded p-summable Fredholm modules, Advances in Math. 151 (2000), 140-163.

[12] A. L. Carey, J. Phillips, F. A. Sukochev, Spectral flow and Dixmier traces, Advances in Math. 173 (2003), 68-113.

[13] A. L. Carey, J. Phillips, A. Rennie, F. A. Sukochev, The Hochschild class of the Chern character of semifinite spectral triples, J. Funct. Analysis, 213 (2004), 111-153.

[14] A. L. Carey, J. Phillips, A. Rennie, APS boundary conditions, KK-theory and spectral flow in graph $C^{*}$-algebras, to appear in Crelle's journal, arXiv:0711.3028.

[15] A. L. Carey, J. Phillips, A. Rennie, Twisted cyclic theory and the modular index theory of Cuntz algebras, to appear in J. K-Theory, arXiv:0801.4605.

[16] A. L. Carey, J. Phillips, A. Rennie, F. A. Sukochev, The local index formula in semifinite von Neumann algebras I, Spectral flow, Adv. Math., 202 (2006), 451-516.

[17] A. L. Carey, J. Phillips, A. Rennie, F. A. Sukochev, The local index formula in semifinite von Neumann algebras II The even case, Adv. Math., 202 (2006), 517-554.

[18] A. L. Carey, J. Phillips, A. Rennie, and F. A. Sukochev, The Chern character of semifinite spectral triples, Journal of Noncommutative Geometry 2 (2008), 141-261.

[19] A. L. Carey, D. S. Potapov, F. A. Sukochev, Spectral flow is the integral of one forms on the Banach manifold of self-adjoint Fredholm operators, to appear in Adv. Math., arXiv:0807.2129.

[20] A. L. Carey, A. Rennie, K. Tong, Spectral flow invariants and twisted cyclic theory from the Haar state on $S U_{q}(2)$, to appear in J. Geom. Phys., arXiv:0802.0317.

[21] A. L. Carey, F. A. Sukochev, Dixmier traces and some applications in noncommutative geometry, UMN, 61 (2006), 45-110.

[22] L.A. Coburn, R.G. Douglas, D.G. Schaeffer, I.M. Singer $C^{*}$-algebras of operators on a half space II: index theory, IHES Publ. Math. 40 (1971), 69-80.

[23] A. Connes, Noncommutative Geometry, Academic Press, 1994.

[24] A. Connes, J. Cuntz, Quasi-homomorphismes, cohomologie cyclique et positivité, Commun. Math. Phys., 114 (1988), 515-526.

[25] A. Connes, H. Moscovici, The local index formula in noncommutative geometry, Geom. Funct. Anal., 5 (1995), 174-243.

[26] A. Connes, H. Moscovici, Transgression du caractère de Chern et cohomologie cyclique, C. R. Acad. Sci. Paris Sr. I Math. 303 (1986), 913-918.

[27] A. Connes, H. Moscovici, Type III and spectral triples, arXiv:math/0609703.

[28] A. Connes, Noncommutative differential geometry, Publ. Math. Inst. Hautes Etudes Sci. (Paris), 62 (1985), 41-44.

[29] A. Connes, Geometry from the spectral point of view, Lett. Math. Phys. 34 (1995), $203-238$.

[30] A. Connes, M. Marcolli, Noncommutative Geometry, Quantum Fields and Motives, American Mathematical Society, Providence RI, 2008.

[31] R. Curto, P.S. Muhly, J. Xia, Toeplitz operators on flows J. Funct. Anal. 93 (1990), 391-450.

[32] T. Fack, H. Kosaki, Generalised s-numbers of $\tau$-measurable operators, Pacific J. Math. 123 (1986), 269-300.

[33] E. Getzler, The odd Chern character in cyclic homology and spectral flow, Topology, 32(1993), 489-507.

[34] E. Getzler, Cyclic homology and the Atiyah-Patodi-Singer index theorem, Index Theory and Operator Algebras, Contemp. Math. 148, Amer. Math. Soc., Providence, RI, (1993), 19-45.

[35] N. Higson, The local index formula in noncommutative geometry, Contemporary Developments in Algebraic K-Theory, ictp Lecture Notes, 15 (2003), 444-536.

[36] A. Jaffe, A. Lesniewski, K. Osterwalder, Quantum K-Theory I. The Chern character, Commun. Math. Phys. 118 (1988), 1-14. 
[37] J. Kaad, R. Nest, A. Rennie, KK-theory and spectral flow in von Neumann algebras, math.OA/0701326.

[38] G. G. Kasparov, The operator K-functor and extensions of $C^{*}$-algebras, Math. USSR. Izv. 16 (1981), 513-572.

[39] M. Lesch, On the index of the infinitesimal generator of a flow, J. Operator Theory 26 (1991), 73-92.

[40] J.-L. Loday, Cyclic Homology 2nd Edition, 1998, Springer- Verlag.

[41] V. Mathai, Spectral flow, eta invariants and von Neumann algebras, J. Funct. Anal., 109 (1992), 442-456.

[42] D. Pask, A. Rennie, The Noncommutative Geometry of Graph $C^{*}$-Algebras I: the Index Theorem, J. Funct. An., 233, (2006), 92-134.

[43] D. Pask, A. Sims, A. Rennie, The Noncommutative Geometry of k-graph $C^{*}$-algebras, J. $K$-Theory, 1, no. 2 (2008), 259-304.

[44] V.S. Perera, Real valued spectral flow in a type $I I_{\infty}$ factor, Ph.D. Thesis, IUPUI, 1993.

[45] V.S. Perera, Real valued spectral flow in a type $I_{\infty}$ factor, preprint, IUPUI, 1993.

[46] J. Phillips, Self-adjoint Fredholm operators and spectral flow, Canad. Math. Bull., 39(1996), 460-467.

[47] J. Phillips, Spectral flow in type I and type II factors-a new approach, Fields Institute Communications, 17(1997), 137-153.

[48] J. Phillips and I. Raeburn, An index theorem for Toeplitz operators with noncommutative symbol space, J. Funct. Anal., 120 (1993), 239-263.

[49] R. Ponge, A new short proof of the local index formula of Atiyah-Singer, in Noncommutative Geometry and Number Theory. Ed. C. Consani and M. Marcolli, Vieweg, Wiesbaden, 2007.

[50] A. Rennie, Smoothness and locality for nonunital spectral triples, K-Theory 28 (2003), $127-161$.

[51] M.A. Shubin, Pseudodifferential almost periodic operators and von Neumann algebras, Trans. Moscow Math. Soc. 1 (1979), 103-166.

[52] C. Wahl, Index theory for actions of compact Lie groups on $C^{*}$-algebras, to appear in J. Operator Theory, arXiv:0707.3207 